\title{
CSF-1-dependant donor-derived macrophages mediate chronic graft-versus-host disease
}

Kylie A. Alexander, ${ }^{1}$ Ryan Flynn, ${ }^{2}$ Katie E. Lineburg, ${ }^{1}$ Rachel D. Kuns, ${ }^{1}$ Bianca E. Teal, ${ }^{1}$ Stuart D. Olver, ${ }^{1}$ Mary Lor, ${ }^{1}$ Neil C. Raffelt, ${ }^{1}$ Motoko Koyama, ' Lucie Leveque, ' Laetitia Le Texier, ${ }^{1}$ Michelle Melino, ${ }^{1}$ Kate A. Markey, ${ }^{1}$ Antiopi Varelias, ${ }^{1}$ Christian Engwerda, ${ }^{1}$ Jonathan S. Serody, ${ }^{3}$ Baptiste Janela, ${ }^{4}$ Florent Ginhoux,${ }^{4}$ Andrew D. Clouston, ${ }^{5}$ Bruce R. Blazar, ${ }^{2}$ Geoffrey R. Hill,,${ }^{1,6}$ and Kelli P.A. MacDonald'

${ }^{1}$ QIMR Berghofer Medical Research Institute, Brisbane, Australia. ${ }^{2}$ Pediatric Blood and Marrow Transplantation Program, University of Minnesota, Minneapolis, Minnesota, USA. ${ }^{3}$ Lineberger Comprehensive Cancer Center, University of North Carolina School of Medicine, Chapel Hill, North Carolina, USA. ${ }^{4}$ Singapore Immunology Network (SIgN), A*STAR (Agency for Science, Technology and Research), Biopolis, Singapore. ${ }^{5}$ Envoi Pathology, Brisbane, Australia. ${ }^{6}$ Department of Bone Marrow Transplantation, Royal Brisbane Hospital, Brisbane, Australia.

\begin{abstract}
Chronic GVHD (cCVHD) is the major cause of late, nonrelapse death following stem cell transplantation and characteristically develops in organs such as skin and lung. Here, we used multiple murine models of cGVHD to investigate the contribution of macrophage populations in the development of cCVHD. Using an established IL-17-dependent sclerodermatous cCVHD model, we confirmed that macrophages infiltrating the skin are derived from donor bone marrow (F4/80+CSF-1 $\left.{ }^{+} \mathrm{CD}_{206}{ }^{+} \mathrm{iNOS}^{-}\right)$. Cutaneous cCVHD developed in a CSF-1/CSF-1R-dependent manner, as treatment of recipients after transplantation with CSF-1 exacerbated macrophage infiltration and cutaneous pathology. Additionally, recipients of grafts from Csf1r/- mice had substantially less macrophage infiltration and cutaneous pathology as compared with those receiving wild-type grafts. Neither CCL2/CCR2 nor GM-CSF/CM-CSFR signaling pathways were required for macrophage infiltration or development of CGVHD. In a different CGVHD model, in which bronchiolitis obliterans is a prominent manifestation, $\mathrm{F} 4 / 80^{+}$macrophage infiltration was similarly noted in the lungs of recipients after transplantation, and lung CCVHD was also IL-17 and CSF-1/ CSF-1R dependent. Importantly, depletion of macrophages using an anti-CSF-1R mAb markedly reduced cutaneous and pulmonary cCVHD. Taken together, these data indicate that donor macrophages mediate the development of cGVHD and suggest that targeting CSF-1 signaling after transplantation may prevent and treat cCVHD.
\end{abstract}

\section{Introduction}

Graft-versus-host disease (GVHD) remains a major complication of allogeneic stem cell transplantation (SCT). GVHD can present in acute and chronic forms, which differ in their time of onset and symptoms. Acute GVHD (aGVHD) occurs early after transplantation, with target organ damage characterized by apoptosis. In contrast, chronic GVHD (cGVHD) is a late complication of SCT and is characterized by fibrosis. Indeed, cGVHD presents with many features that overlap with certain autoimmune diseases. While skin is the primary organ involved in cGVHD, both lung and liver fibrosis can also occur, and these manifestations are all associated with significant morbidity and mortality. Our understanding of the pathophysiology of aGVHD is far more advanced than that of cGVHD, and it is clear that these forms are mediated by different immunological subsets and cytokine networks. Currently, the majority of clinical allogeneic trans-

Note regarding evaluation of this manuscript: Manuscripts authored by scientists associated with Duke University, The University of North Carolina at Chapel Hill, Duke-NUS, and the Sanford-Burnham Medical Research Institute are handled not by members of the editorial board but rather by the science editors, who consult with selected external editors and reviewers.

Conflict of interest: The authors have declared that no conflict of interest exists Submitted: March 4, 2014; Accepted: July 10, 2014.

Reference information: / Clin Invest. 2014;124(10):4266-4280. doi:10.1172/JCI75935. plants use granulocyte colony-stimulating factor-mobilized (G-CSF-mobilized) peripheral blood (PB) stem cells, which has led to rapid hematopoietic reconstitution, improved leukemic eradication, and similar levels of aGVHD, but increased levels of cGVHD (1). Therapeutically, there are limited options available for the treatment of cGVHD, with corticosteroids representing the mainstay of treatment. Unfortunately, this is often ineffective and is associated with significant morbidity, thus cGVHD represents an increasing burden in the clinic.

Macrophages play an essential role in both homeostasis and pathology and are characterized by high functional heterogeneity (2). The differentiation, proliferation, and survival of the majority of macrophage populations are dependent on colony-stimulating factor 1 (CSF-1) (3, 4). Macrophages acquire diverse phenotypic and functional forms in response to local cytokines and microbial signals, which has resulted in the "M1" and "M2" macrophage classifications $(5,6)$. Classically activated macrophages (M1) mediate defense of the host from a variety of bacteria, protozoa, and viruses and have roles in antitumor immunity. Activation of proinflammatory M1 macrophages is typically induced by IFN- $\gamma$, lipopolysaccharide, and cytokines such as granulocyte-macrophage colony-stimulating factor (GM-CSF) (5-7). Alternatively activated macrophages (M2) have antiinflammatory functions and regulate tissue repair and remodeling. M2 macrophage activation is induced by IL-4 and IL-13 (8) as well as immune complexes, glucocorticoids, and the 
GCSF Balb/c $\rightarrow$ B6

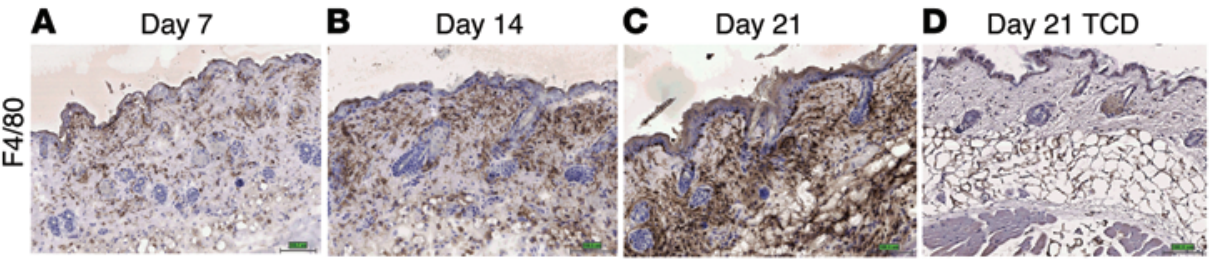

$\mathrm{C} 57 \mathrm{BI} / 6 \rightarrow \mathrm{B} 6 \mathrm{D} 2 \mathrm{~F} 1$
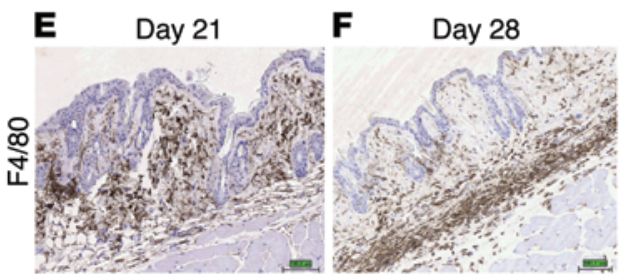

G Day 35

H Day $35 \mathrm{TCD}$

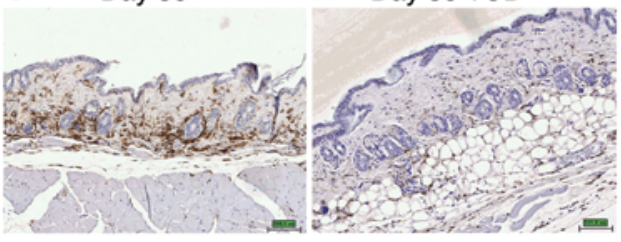

Figure 1. $\mathrm{F} 4 / \mathbf{8 0}^{+}$macrophages infiltrate the skin of mice receiving either mobilized or nonmobilized grafts. (A-H) IHC to detect F4/80 expression in recipients of mobilized and nonmobilized grafts. Representative images of skin from lethally irradiated B6 mice that received G-CSF-mobilized BALB/c grafts. IHC was performed on days 7 (A), 14 (B), and 21 (C) after transplantation ( $n=3 /$ time point). F4/80+ macrophages were present in the dermis by day 7 after transplantation $(\mathbf{A})$, with robust infiltration throughout the dermis at day 14 (B) and subcutaneous fat from day 21 (C). (D) Minimal F4/80+ macrophage infiltrate was noted in the skin of mice that received TCD grafts. (E-H) Representative images of F4/80 IHC in lethally irradiated B6D2F1 mice that received B6 BM plus T cell grafts. Skin was examined on days 21 (E) , 28 (F), and 35 (G) after transplantation ( $n=3 /$ time point). F4/80+ macrophage infiltrate was present in the dermis from day 21 after transplantation (E) and throughout the subcutaneous fat layer by day 28 ( $\mathbf{F}$ and $\mathbf{G})$. There was minimal F4/80+ macrophage infiltrate in mice that received TCD grafts $(\mathbf{H})$. Original magnification, $\times 5$. opment of CGVHD, and this was therefore the focus of this study.

\section{Results}

$\mathrm{F} / 8 \mathrm{O}^{+}$macrophages infiltrate the skin of mice receiving either G-CSF-mobilized or -nonmobilized grafts. We investigated the kinetics of macrophage infiltration into the skin after transplantation in lethally irradiated mice receiving either allogeneic, G-CSF-mobilized $(\mathrm{G}-\mathrm{CSF}$ BALB/c graft $\rightarrow \mathrm{C} 57 \mathrm{Bl} / 6$ [B6] recipients; Figure 1, A-D), or semiallogeneic, nonmobilized grafts (B6 bone marrow [BM] plus $\mathrm{T}$ cell graft $\rightarrow$ B6D2F1 recipients; Figure 1, E-H) that develop sclerodermatous cGVHD. IHC analysis for $\mathrm{F} 4 / 80$ expression demonstrated that $\mathrm{F} 4 / 80^{+}$macrophages infiltrated the dermis of mice receiving G-CSF-mobilized grafts starting 7 days after transplantation (Figure 1, $\mathrm{A}$ and $\mathrm{B}$ ), with robust $\mathrm{F} 4 / 80^{+}$macrophage infiltration present by day 21 after transplantation throughout the dermis and s.c. fat layers (Figcytokine CSF-1 $(6,7)$. Although the M1 and M2 classification system is widely used, it is increasingly clear that these macrophage populations represent the extreme ends of a wide spectrum of phenotypes associated with macrophage activation.

Recent preclinical and clinical data from our group established a highly reproducible and informative model of cGVHD that supports a role for IL-17 as a central mediator of pathology, particularly within the skin (9). Cutaneous cGVHD was shown to be exacerbated by G-CSF mobilization in an IL-17-dependent manner, and late after transplantation, scleroderma was absent in recipients of $I l 17 a^{-/-}$grafts compared with wild-type (WT) grafts. Interestingly, IL-17A controlled the infiltration of $\mathrm{F} 4 / 80^{+}$macrophages into skin, and this preceded the development of scleroderma, suggesting that macrophages play important profibrotic roles in the development of cutaneous cGVHD.

There is an expanding body of evidence suggesting that macrophages can play both anti- $(10,11)$ and profibrotic roles $(12,13)$. To date, models of macrophage depletion in vivo have been unsatisfactory, inducing rapid cell death and inflammation that complicate experimental conclusions. Nevertheless, studies in renal (13), hepatic $(10)$, lung $(12)$, and skin fibrosis $(14,15)$ suggest that macrophage depletion can modify fibrosis. Cutaneous macrophage infiltration has been suggested to be associated with refractory aGVHD and poor prognosis (16). Alternatively, activated macrophages $\left(\mathrm{CD} 163^{+}\right)$have been previously shown to be present in both humans (17) and rats with systemic sclerosis (18). Additionally, macrophages displaying an M2 phenotype are present in the skin (19) and lungs of patients with systemic sclerosis (20). However, there are no studies to date that demonstrate a causal role of macrophages in the devel- ure 1C). We noted minimal macrophage infiltration in mice that received G-CSF-mobilized T cell-depleted (TCD) grafts (Figure 1D). In mice receiving nonmobilized grafts, we noted $\mathrm{F} 4 / 80^{+}$macrophage infiltration in the dermis by day 21 after transplantation (Figure 1E), and by day 28 after transplantation, $\mathrm{F} 4 / 80^{+}$macrophages were predominantly located within the s.c. fat layer (Figure $1, F$ and $G$ ), with minimal macrophage infiltration noted in mice that received TCD grafts (Figure $1 \mathrm{H}$ ). Although not examined here, our initial studies also identified neutrophils and $\mathrm{T}$ cells within the mononuclear infiltrate in skin during GVHD (9), however, they were far less dominant than the $\mathrm{F} 4 / 80^{+}$macrophage infiltrate illustrated in Figure 1, and only the macrophages were IL-17 dependent.

Skin-infiltrating macrophages are donor M2-like CSF-1Rexpressing macrophages. To examine the phenotype of these $\mathrm{F} 4 / 80^{+}$macrophages, IHC was performed using markers for both classically activated and alternatively activated macrophages (inducible nitric oxide synthase [iNOS] and mannose receptor CD206, respectively). Additionally, IHC for the congenic marker CD45.1 was used to mark donor cells. IHC in serial sections was performed 21 days after transplantation in lethally irradiated mice receiving either G-CSF-mobilized non-TCD grafts (cGVHD mice) or G-CSF-mobilized TCD grafts (no cGVHD controls). Results confirmed that the vast majority of infiltrating $\mathrm{F} 4 / 80^{+}$cells in mice receiving G-CSF-mobilized grafts were donor (CD45.1 ${ }^{+}$) alternatively activated $\left(\mathrm{CD} 206^{+}\right)$macrophages, with minimal iNOS expression $\left(\mathrm{F} 4 / 80^{+} \mathrm{CD} 45.1^{+} \mathrm{CD}^{206}{ }^{+}{ }^{+} \mathrm{NOS}^{-}\right)$(Figure $2 \mathrm{~A}$ and Supplemental Figure 1A; supplemental material available online with this article; doi:10.1172/JCI75935DS1). We noted minimal donor macrophage infiltration in mice that received G-CSF-mobilized 
A

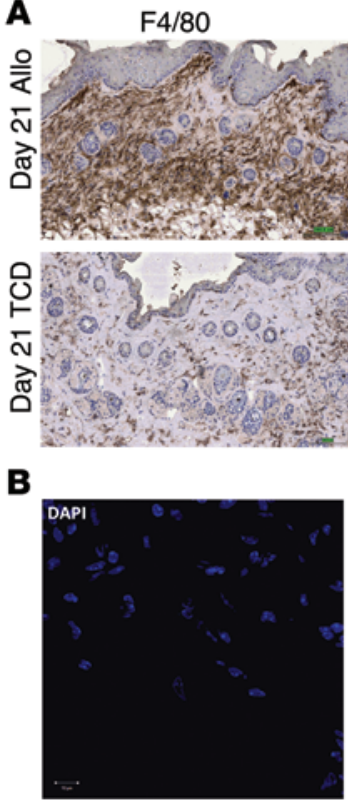

CD45.1 (donor)
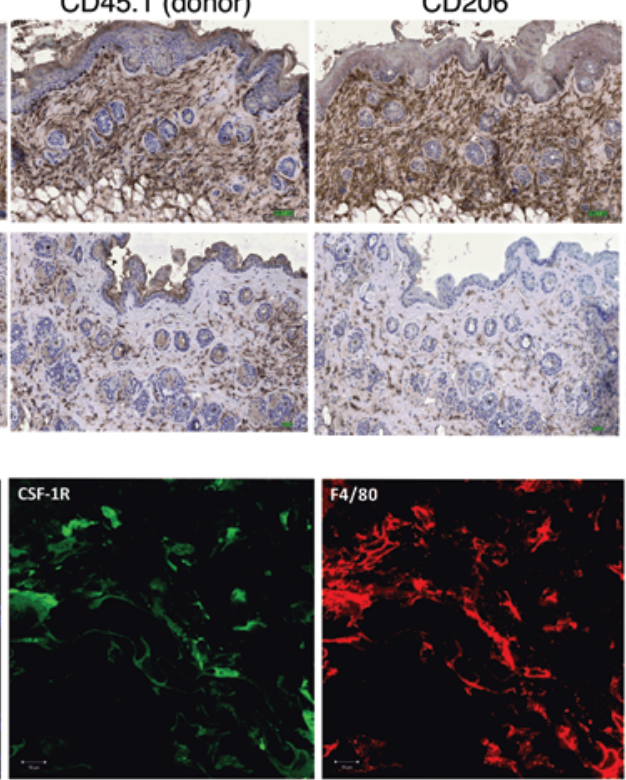

Figure 2. $\mathbf{F 4 / 8 0 ^ { + }}$ macrophages infiltrating the skin after transplantation are donor M2-like macrophages that express CSF-1R. (A) Representative IHC images from lethally irradiated B6 mice that received G-CSF-mobilized BALB/C (CD45.1) or G-CSF-mobilized TCD grafts. IHC to analyze F4/80, CD45.1, CD206, and iNOS expression illustrates that at day 21 after transplantation, donor M2-like macrophages were present within the skin ( $\mathrm{F} 4 / 80^{+}$, $\mathrm{CD}_{45} 1^{+}, \mathrm{CD}^{206^{+}}$, and iNOS-). Minimal donor M2-like macrophage infiltrate was detected in mice that received TCD grafts. Original magnification, $\times 5$. (B) Representative IF images 28 days after transplantation from skin of lethally irradiated B6D2F1 mice that received BM plus T cell grafts from MacGreen transgenic mice (Csf1r promoter driving EGFP). $n=3$ /time point. Original magnification, $\times 20$. IF confirmed that CSF-1R+ cells (green) were also F4/80+ (red); white arrows.

TCD grafts. We also noted alternatively activated macrophage infiltrate in lethally irradiated mice that received nonmobilized grafts (data not shown). B6D2F1 mice receiving BM plus $\mathrm{T}$ cell grafts from MacGreen mice (MacGreen mice have EGFP driven off the Csflr promoter) (21) confirmed that these $\mathrm{F} 4 / 80^{+}$macrophages are also CSF-1 $\mathrm{R}^{+}$(Figure 2B). Using the same transplantation system, we additionally confirmed that donor $\left(\mathrm{CSF}-1 \mathrm{R}^{+}\right)$cells infiltrate the skin as early as 7 days after transplantation (Supplemental Figure $2 \mathrm{~A}$ ), and by day 21 , the vast majority of $\mathrm{F} 4 / 80^{+}$cells are of donor origin (Supplemental Figure 2B).

Cytokine/chemokine dependency of blood monocyte populations in naive mice. Current dogma holds that circulating monocytes can give rise to a variety of tissue-resident macrophages throughout the body, particularly during inflammation. In the steady state, circulating $\mathrm{CD} 11 \mathrm{~b}^{+} \mathrm{F} 4 / 80^{+}$monocytes can be divided into 2 distinct cell populations based on the expression of Ly6C (22). CD11b $\mathrm{F} 4 / 80^{+} \mathrm{Ly}_{6} \mathrm{C}^{\mathrm{hi}}$ monocytes express the C-C chemokine receptor type 2 (CCR2) and are commonly referred to as classical or inflammatory monocytes. In contrast, $\mathrm{CD} 11 \mathrm{~b}^{+} \mathrm{F} 4 / 80^{+} \mathrm{Ly} 6 \mathrm{C}^{\text {lo }}$ monocytes are CCR2-, but express the fractalkine receptor CX3CR1 and are commonly referred to as patrolling monocytes, which are widely considered to be tissue-resident macrophage precursors. To elucidate the monocyte precursors and cytokine dependency of the donor infiltrating macrophages following BM transplantation (BMT), we next examined the cytokine and chemokine requirements for the development and migration of the distinct PB monocyte populations. For these studies, we examined both frequency (percentage) and enumer-
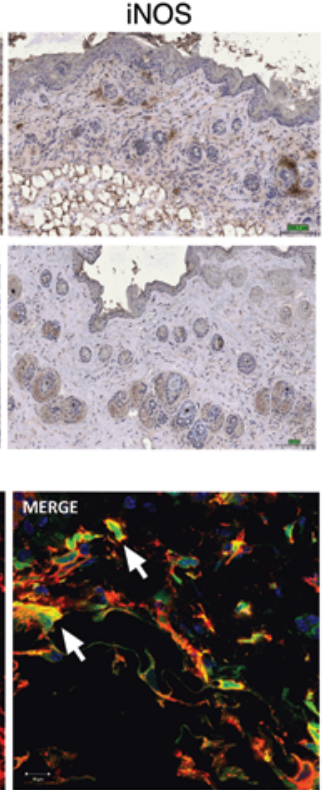

ated PB monocyte populations in naive B6.WT, B6.Ccr2 ${ }^{-}$, and $\mathrm{B} 6$ common $\beta$ chain $^{-/}$mice to examine the requirement of chemokine (C-C motif) ligand 2 (CCL2) and GM-CSF, respectively. To examine the requirement of CSF-1, we used fetal liver chimeras (FLCs) on a $\mathrm{B} 6$ background, since CSF-1R deficiency is neonatally lethal. We examined monocyte populations using a gating strategy (Figure $3 \mathrm{~A})$ that removed $\mathrm{Ly} 6 \mathrm{G}^{+} \mathrm{CD}^{+}$and high side-scatter cells (SSCs). As expected, compared with B6.WT mice, we observed a significant reduction in the frequency and absolute numbers of circulating $\mathrm{CD} 11 \mathrm{~b}^{+} \mathrm{F} 4 / 80^{+} \mathrm{Ly}_{6 \mathrm{C}} \mathrm{Ci}^{\mathrm{i}} \quad$ classical monocytes in the $\mathrm{Ccr}^{2-}$ mice (Figure 3, B and $\mathrm{C} ;{ }^{* *} \mathrm{P}=0.004$, $\left.{ }^{*} P=0.0081\right)$. In contrast, compared with B6.WT FLCs, we found that the $\mathrm{B} 6 \mathrm{Csf1}{ }^{\circ}$ FLCs exhibited a significant reduction in $\mathrm{Ly}^{6} \mathrm{C}^{\mathrm{lo}}$ subset frequencies and absolute numbers (Figure 3, B and C; $\left.{ }^{* * *} P=0.002,{ }^{*} P=0.0286\right)$. The contribution of GM-CSF to circulating monocyte populations was minimal, since the frequencies and absolute numbers of both Ly $6 \mathrm{C}^{\mathrm{hi}}$ and $\mathrm{Ly} 6 \mathrm{C}^{\mathrm{lo}}$ cell populations were not significantly altered in the common $\beta$ chain-deficient mice.

CSF-1 treatment after SCT exacerbates cutaneous cGVHD. We had previously confirmed in Figure 3 that CSF-1 was required for the development of the Ly6 $6 \mathrm{C}^{\text {lo }} \mathrm{PB}$ monocyte/macrophage population, which is suggested to be a tissue macrophage precursor. Therefore, we further examined the contribution of CSF-1 to macrophage infiltration and cutaneous CGVHD after transplantation. Lethally irradiated B6 mice received G-CSF-mobilized BALB/c whole spleen or TCD spleen and were treated with either saline or CSF-1 for 5 days from day 14 after transplantation. Compared with saline-treated recipients, we found that CSF-1 treatment significantly increased $\mathrm{F} 4 / 80^{+}$macrophages in the skin 19 days after transplantation (Figure 4A), quantified as positive pixels/ $\mathrm{mm}^{2}$ (Figure $4 \mathrm{~B} ;{ }^{* *} P=0.0017$ ). CSF-1 treatment preferentially expanded donor alternatively activated macrophages, as confirmed via IHC (F4/80 $\left.{ }^{+} \mathrm{CD} 45.1^{+} \mathrm{CD}^{206} 6^{+} \mathrm{NNOS}^{-}\right)$(Figure 4C). Semiquantitative histopathology confirmed that recipients treated with CSF-1 had significantly higher cutaneous pathology scores than did those treated with saline (Figure $4 \mathrm{D} ;{ }^{* *} P=0.0087$ ).

Further analysis of PB monocyte populations 19 days after transplantation illustrated a trend toward an increase in the frequency of Ly6C $\mathrm{C}^{\text {lo }}$ cells after 5 days of CSF-1 treatment (Figure $5 \mathrm{~A})$. Therefore, we carried out further monocyte/macrophage analysis of cells isolated 19 days after transplantation from the skin (ears) of mice treated with either saline or CSF-1 (gating 


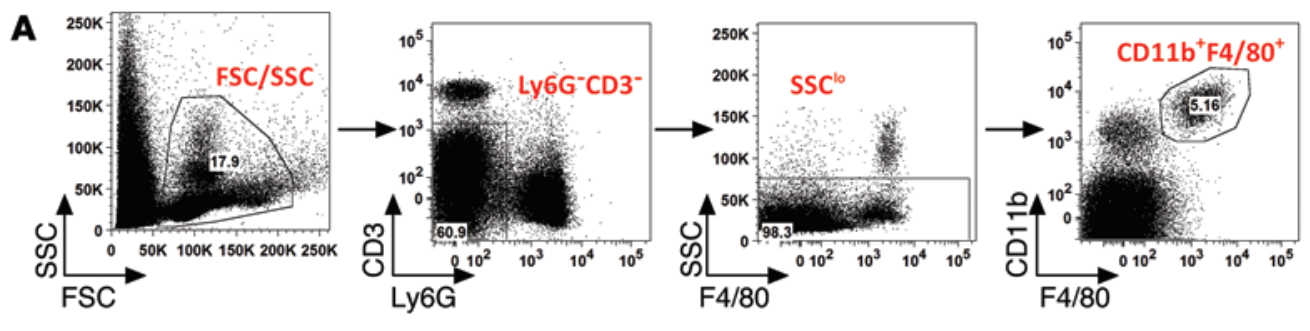

$\mathbf{B}$
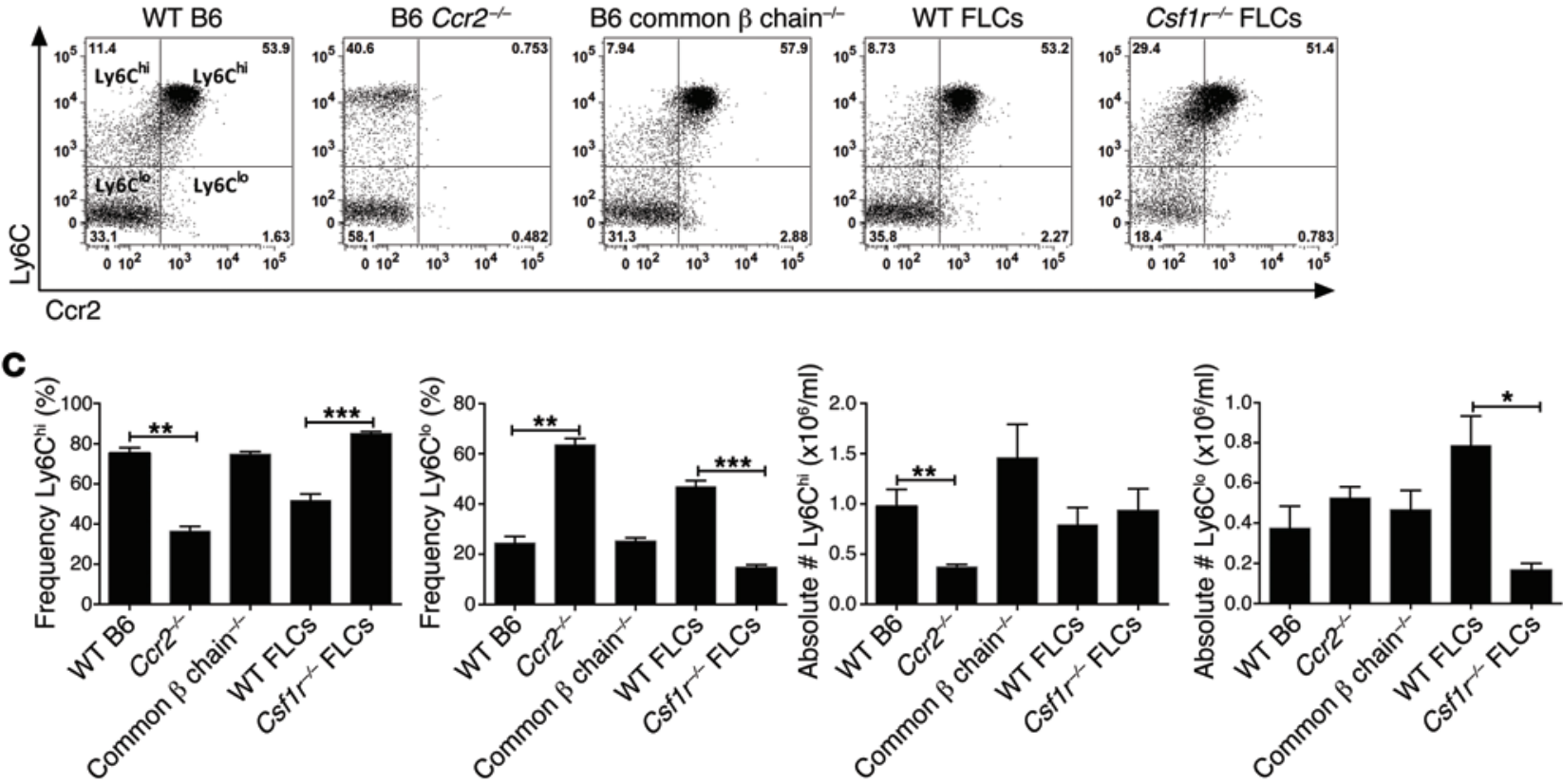

Figure 3. Cytokine/chemokine dependency of PB monocyte populations in naive mice. (A) Representative dot plots illustrate the gating strategy for PB monocytes and macrophages. Numbers in each dot plot indicate the percentage of positive cells in each gate. (B) Analysis of PB monocytes and macrophages from B6, $\mathrm{Cr}^{-1-}$, and common $\beta \mathrm{chain}^{-1-}$ mice as well as from WT and $\mathrm{Csf1r^{-1- }}$ FLCs. Numbers in each dot plot indicate the percentage of Ly6C ${ }^{\mathrm{hi}}$ cells (top 2 quadrants) and Ly6 $C^{10}$ cells (bottom 2 quadrants) and their expression of CCR2. (C) Frequency (percentage) and absolute numbers ( $\left.\times 10^{6} / \mathrm{ml}^{\prime}\right)$ of Ly6C ${ }^{\text {hi }}$ versus Ly $6 C^{10}$ monocyte and macrophage populations in naive mice (frequency of Ly6C $C^{\text {hi }}$ cells: ${ }^{* *} P=0.004,{ }^{* * *} P=0.0002$; frequency of Ly6C ${ }^{10}$ cells: ${ }^{* *} P=0.004,{ }^{* * *} P=0.0002$; absolute numbers of Ly6Chi cells: ${ }^{* *} P=0.0081$; absolute numbers of Ly6C ${ }^{10}$ cells: ${ }^{*} P=0.0286$ ). $n=8$ for all groups. Statistically significant differences were calculated using 2-tailed Mann-Whitney $U$ tests. Data represent the mean \pm SEM.

strategy for monocyte/macrophage analysis; Figure 5B). We performed analysis using a gating strategy similar to that shown in Figure 3A, with monocytes (Figure 5B, Mono) being classified as Ly6 $\mathrm{C}^{\text {lo }} \mathrm{SSC} \mathrm{C}^{\text {lo }} \mathrm{F} 4 / 8 \mathrm{O}^{+} \mathrm{CD} 11 \mathrm{~b}^{+}$and macrophages (Figure $5 \mathrm{~B}, \mathrm{M} \phi$ ) as Ly6 $\mathrm{C}^{\mathrm{lo}} \mathrm{SSC} \mathrm{C}^{\text {hi }} \mathrm{F} 4 / 80^{+}$. Data are expressed as the absolute number of $\mathrm{CD} 45^{+}$cells for both monocytes and macrophages (Figure 5C). We observed a significant increase in absolute numbers in both the $\mathrm{CD}^{+} 5^{+}$monocyte population $\left(\mathrm{Ly} 6 \mathrm{C}^{\mathrm{lo}} \mathrm{CD} 11 \mathrm{~b}^{+} \mathrm{F} 4 / 80^{+}\right.$) (Figure $5 \mathrm{C}$; ${ }^{* *} \mathrm{P}=0.0087$ ) and $\mathrm{CD} 45^{+}$macrophage population $\left(\mathrm{Ly} 6 \mathrm{C}^{\mathrm{lo}} \mathrm{F} 4 / 80^{+}\right)$ (Figure 5C; ${ }^{*} P=0.026$ ) in the CSF-1-treated mice compared with the absolute numbers detected in saline-treated mice. Overall, this confirmed that CSF-1 treatment after transplantation significantly increased cutaneous $\mathrm{Ly}^{6} \mathrm{C}^{\mathrm{lo}}$ monocytes and macrophages compared with those detected in saline-treated recipients.

F4/80 macrophage infiltration and cutaneous fibrosis after transplantation is CSF-1R dependent. We subsequently investigated the requirement for donor CSF-1R signaling in the development of cutaneous cGVHD using WT and Csfir $/$ FLCs. Lethally irradiated $\mathrm{B} 6 \mathrm{D} 2 \mathrm{~F} 1$ recipients were given $\mathrm{BM}$ plus $\mathrm{T}$ cell grafts from either $\mathrm{B} 6$ WT FLC, CsfIr ${ }^{-}$FLC, or WT FLC TCD grafts. Mice that received
Csflr $r$ grafts had a significantly lower clinical GVHD score throughout the time course examined compared with that of WT graft recipients (Figure 6A). IHC performed 21 days after transplantation illustrated that mice receiving WT grafts (Figure 6B) had significantly higher $\mathrm{F} 4 / 80^{+}$macrophage infiltration compared with that seen in mice receiving $\mathrm{Csfl}^{\circ}-$ grafts (Figure 6B), quantified as positive pixels $/ \mathrm{mm}^{2}$ (Figure $6 \mathrm{C} ;{ }^{* *} P=0.003$ ). Masson's trichrome staining (collagen fibers are stained blue) and semiquantitative histopathology scores assessed 48 days after transplantation confirmed that mice receiving $\mathrm{Csfl}^{-/}$grafts had significantly lower cutaneous pathology and fibrosis than did those receiving WT grafts (Figure $6 \mathrm{D} ;{ }^{*} P=0.045,{ }^{* *} P=0.008$ ), confirming a pathogenic role for CSF-1/CSF-1R signaling in the development of cutaneous pathology after transplantation. Analysis of PB monocyte populations 21 days after transplantation illustrated that recipients of $\mathrm{Csfl}^{\circ}{ }^{/-} \mathrm{FLC}$ grafts exhibited a significant increase in the frequency of Ly $6 \mathrm{C}^{\text {hi }}$ cells and a significant reduction in the Ly $6 \mathrm{C}^{\text {lo }}$ cell population (Figure 6E; $\left.{ }^{*} P=0.0286\right)$. Other CSF-1R-dependent cells reside within the skin apart from macrophages, most notably langerin-expressing Langerhans cells, which reside in the epidermis. In contrast to macrophages, 
A
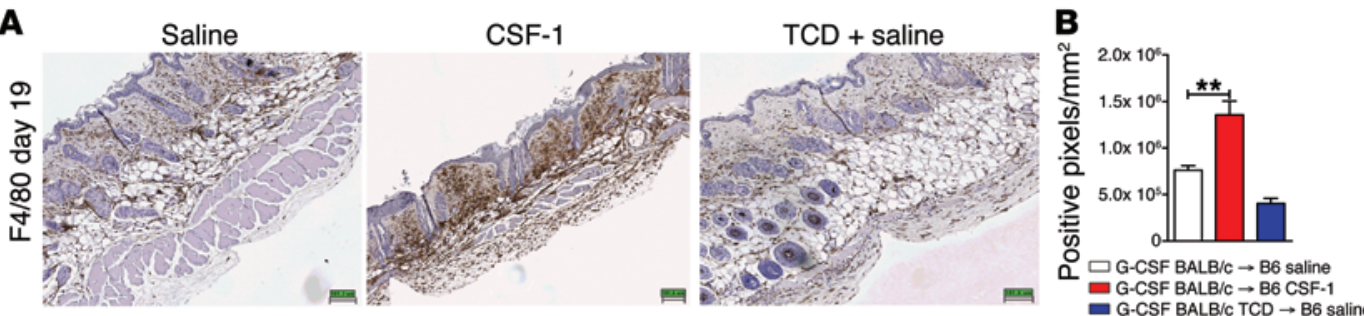

C

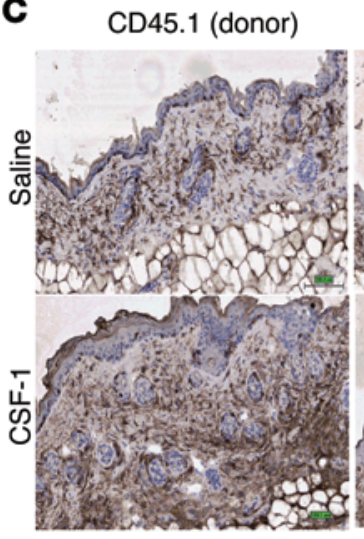

$\mathrm{F} 4 / 80$

CD206
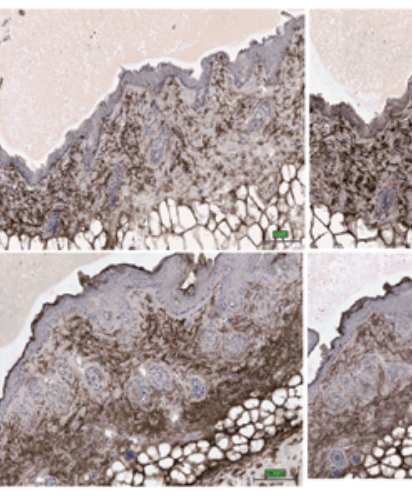

iNOS
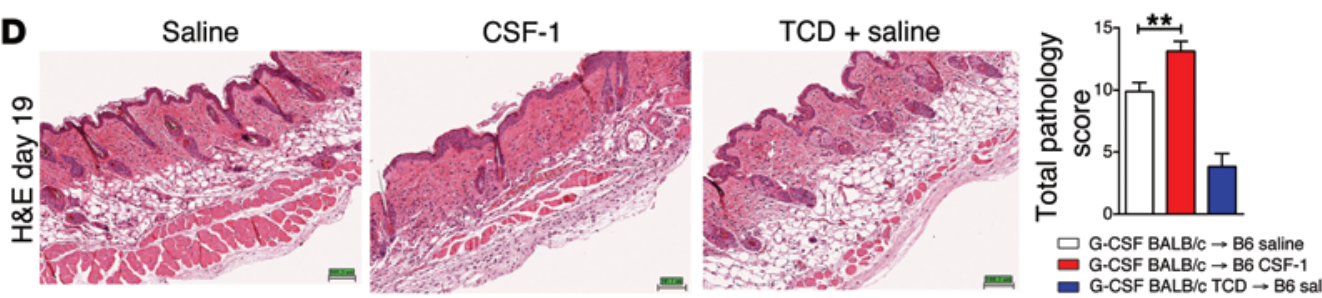

Figure 4. CSF-1 treatment after SCT exacerbates cutaneous CGVHD. Lethally irradiated B6 mice received G-CSF-mobilized WT BALB/C (CD45.1) or TCD grafts and were treated with either saline or CSF-1 (10 $\mu \mathrm{g} /$ daily) from days 14 to 19 after transplantation. (A) Representative IHC images of F4/80 expression in the skin of saline- and CSF-1-treated mice. CSF-1 treatment resulted in a significant increase in $\mathrm{F} 4 / 80^{+}$cells within the skin compared with cells detected in saline-treated control mice, quantified in B as positive/pixels $\left./ \mathrm{mm}^{2}{ }^{* *} P=0.007\right)$. (C) CSF-1 treatment preferentially expanded donor M2 macrophages in the skin (CD45.1 $1^{+}$[donor], $\mathrm{F} 4 / 80^{+}, \mathrm{CD}^{206}{ }^{+}$, and $\mathrm{iNOS}^{-}$) compared with levels detected in saline control mice. (D) Representative H\&E-stained images and semiquantitative histopathology ( $n=8 /$ group for all groups, except 6/group for TCD) illustrating that (SF-1 treatment resulted in a significantly higher cutaneous pathology score compared with that for saline-treated mice $\left({ }^{* *} P=0.006\right)$. Statistically significant differences were calculated using 2-tailed Mann-Whitney $U$ tests. Data represent the mean \pm SEM. Original magnification, $\times 5$ (A and $\mathbf{B})$ and $\times 8$ (C).

tion of GM-CSF signaling to macrophage infiltration and cutaneous GVHD. Serum GM-CSF was only detected 7 days after transplantation (Figure 7A; ${ }^{*} P=0.01$ ), with no difference in serum GM-CSF levels between mice receiving either allogeneic or TCD grafts from day 14 after transplantation. $\mathrm{F} 4 / 80^{+}$macrophages were still noted 21 days after transplantation in the skin of lethally irradiated mice that received WT or common $\beta$ chain $^{-1-}$ BM plus $\mathrm{T}$ cell grafts (Figure $7 \mathrm{~B}$ ). The common $\beta$ chain $^{-1}$ mouse has defective signaling for IL-3 and IL-5 as well as for GM-CSFR signaling (24), therefore, we specifically blocked GM-CSFR signaling with an anti-GM-CSF Ab (M250 mAb; Amgen). Treatment with M250 starting 7 days after transplantation had no effect on $\mathrm{F} 4 / 80^{+}$macrophage infiltration (Figure 7C), quantified as positive pixels $/ \mathrm{mm}^{2}$ (Figure 7D). PB monocyte analysis 21 days after transplantation showed no change in the frequencies of $\mathrm{Ly} 6 \mathrm{C}^{\mathrm{hi}}$ and

however, Langerhans cells are instructed by the alternative CSF-1R ligand IL-34 (23). To investigate the contribution of Langerhans cells to the development of CSF-1R-dependent sclerodermatous cGVHD, mice received $\mathrm{BM}$ and $\mathrm{T}$ cell grafts from langerin-diphtheria toxin receptor-transgenic (langerin-DTR-transgenic) mice in which DTR and EGFP are expressed under the control of the $\mathrm{Cd} 207$ (encoding langerin) promoter. Administration of DT between 7 and 40 days after transplantation effectively depleted donor Langerhans cells (Supplemental Figure 3A), but neither $\mathrm{F} 4 / 80^{+}$macrophage infiltration nor the development of cutaneous pathology was altered (Supplemental Figure 3, B and C), thus establishing that Langerhans cells do not contribute to cutaneous CGVHD.

F4 $/ 80^{+}$macrophage infiltration and cutaneous fibrosis after transplantation is GM-CSFR independent. Both CSF-1/CSF-1R and GM-CSF/GM-CSFR signaling pathways are involved in regulating the number and function of macrophage lineage populations, and both signaling pathways have been shown to contribute to macrophage heterogeneity (7). Therefore, we examined the contribu-
Ly $6 \mathrm{C}^{\text {lo }}$ cell populations in mice receiving either $\mathrm{B} 6 \mathrm{WT}$ or $\mathrm{B} 6 \mathrm{com}$ mon B chain ${ }^{--}$grafts (Figure 7E). Thus, GM-CSF is not required for the infiltration of $\mathrm{F} 4 / 80^{+}$macrophages and the subsequent development of cutaneous pathology after transplantation.

Development of cutaneous pathology is CCR2 independent. Monocyte chemoattractant protein 1 (MCP-1/CCL2), a multifunctional chemokine belonging to the $\mathrm{C}^{-} \mathrm{C}$ chemokine superfamily, has been shown to be upregulated in a variety of fibrotic conditions (25-27). Furthermore, patients with scleroderma exhibit elevated levels of CCL2 in both serum and skin $(28,29)$. Similarly, we found elevated levels of CCL2 in the serum from 7 to 28 days after transplantation in lethally irradiated mice receiving BM plus T cell grafts (Allo) compared with levels detected in TCD non-GVHD controls (Figure 8A). To investigate the contribution of donor CCL2/CCR2 signaling to the development of cutaneous GVHD, lethally irradiated mice received BM from B6 WT or B6 $\mathrm{Ccr} 2^{-/-}$mice with B6 WT T cells. IHC demonstrated that $\mathrm{F} 4 / 80^{+}$ macrophages were still present in the skin 28 days after transplan- 
A

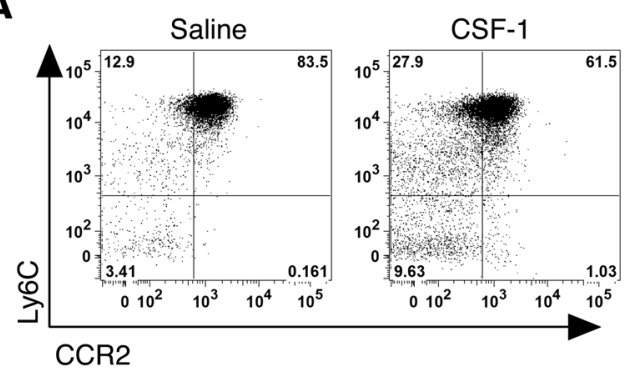

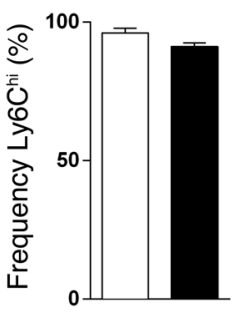

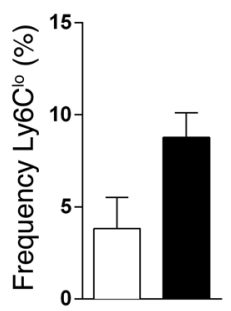

$\square$ G-CSF BALB/C $\rightarrow$ B6 saline G-CSF BALB/C $\rightarrow$ B6 CSF-1

\section{B}

Gated on live, CD45+, singlet cells:
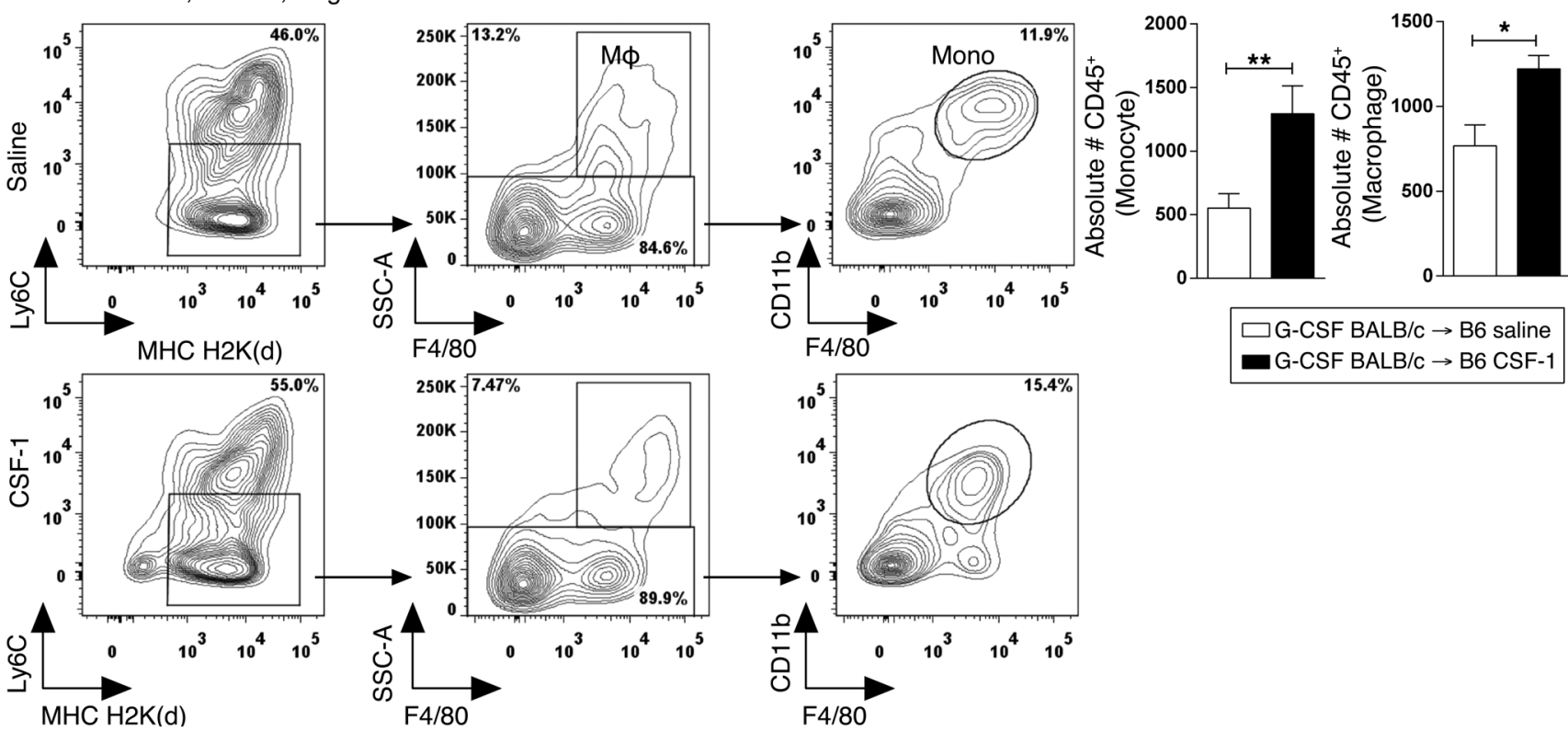

G-CSF BALB/C $\rightarrow$ B6 CSF-1

Figure 5. Analysis of PB and cutaneous monocyte and macrophage populations after transplantation and CSF-1 treatment. (A) Representative dot plots of PB monocyte and macrophage analysis of recipients 19 days after transplantation. Numbers in each dot plot indicate the percentage of Ly6Chi cells (top 2 quadrants) and Ly6 $\mathrm{C}^{10}$ cells (bottom 2 quadrants) and their expression of CCR2. Results illustrate a trend toward increased frequencies of Ly6 ${ }^{10}$ cells after CSF-1 treatment. Representative dot plot shows frequency of Ly6Chi cells (saline, 96.4\% and CSF-1, 89.4\%) and Ly6C ${ }^{10}$ cells (saline, 3.57\% and CSF-1, $10.66 \%$ ). (B) Representative dot plots illustrate the gating strategy for cutaneous monocyte and macrophage analysis of recipients 19 days after transplantation. Numbers in each dot plot indicate the percentage of positive cells in each gate. Results illustrate a significant increase in the absolute numbers of CD45 monocytes (Ly6C $\left.{ }^{10} \mathrm{CD} 11 \mathrm{~b}^{+} \mathrm{F} 4 / 80^{+}\right)$and $\mathrm{CD} 45^{+}$macrophages (Ly6C $\left.{ }^{10} \mathrm{~F} 4 / 80^{+}\right)$. CD45+Ly6C ${ }^{10} \mathrm{CD} 11 \mathrm{~b}^{+} \mathrm{F} 4 / 80^{+}$monocyte absolute numbers: ${ }^{* *} P=0.0087$; CD45 ${ }^{+}$Ly $6 C^{10} \mathrm{~F} 4 / 80^{+}$macrophage absolute numbers: ${ }^{*} P=0.026$ ( $n=6 /$ group, from 3 mice/group; 2 ears/mouse). Statistically significant differences were calculated using 2-tailed Mann-Whitney $U$ tests. Data represent the mean $\pm \mathrm{SEM}$.

tation in mice receiving either B6 WT or B6 $\mathrm{Ccr}^{-/}$grafts (Figure $8 \mathrm{~B}$ ), quantified as positive pixels $/ \mathrm{mm}^{2}$ (Figure $8 \mathrm{C}$ ). We also observed no difference in cutaneous pathology in the recipients of WT or $\mathrm{Crr}^{-/}$grafts (Figure 8, D and E). Similarly, Ab-mediated blockade using an anti-CCL2 Ab had no significant effect on $\mathrm{F} 4 / 80^{+}$macrophage infiltration (Figure $8, \mathrm{~F}$ and $\mathrm{G}$ ) or subsequent cutaneous pathology (Figure $8 \mathrm{H}$ ). $\mathrm{PB}$ monocyte analysis 21 days after transplantation illustrated no change in the frequencies of Ly6 $\mathrm{C}^{\text {hi }}$ or Ly $6 \mathrm{C}^{\text {lo }}$ cell populations in mice receiving either B6 WT or $\mathrm{B} 6 \mathrm{Crr}^{-/}$grafts (Figure 8I). Thus, CCL2/CCR2 signaling is not required for the infiltration of $\mathrm{F} 4 / 80^{+}$macrophages and the subsequent development of cutaneous pathology after transplantation. This suggests that the chemokine CCL2 is redundant for monocyte recruitment in the context of the GVHD inflammatory milieu.

Anti-CSF-1R Ab treatment after transplantation attenuates cutaneous GVHD. To investigate whether depletion of the CSF-1/
CSF-1R-dependent macrophage population after transplantation was an effective therapeutic strategy to prevent and treat cGVHD, we used an anti-CSF-1R Ab (M279; Amgen). We have previously demonstrated that M279 specifically depletes tissue-resident macrophages (30). Lethally irradiated B6 recipients of G-CSFmobilized $\mathrm{BALB} / \mathrm{c}$ grafts were treated with either rat $\operatorname{IgG}$ control or M279 mAb beginning 7 days after transplantation. IHC staining demonstrated that M279 mAb treatment significantly depleted $\mathrm{F} 4 / 80^{+}$macrophages in the skin compared with levels detected rat IgG-treated control mice (Figure 9A), quantified as positive pixels $/ \mathrm{mm}^{2}$ (Figure 9B; ${ }^{* * *} P=0.002$ ). Long-term depletion (out to day 48) of $\mathrm{F} 4 / 80^{+}$macrophages in $\mathrm{B} 6 \mathrm{D} 2 \mathrm{~F} 1$ mice that received $\mathrm{B} 6 \mathrm{BM}$ and $\mathrm{T}$ cells or TCD grafts also confirmed that M279 mAb treatment significantly reduced cutaneous GVHD (Figure 9, C and $\mathrm{D} ;{ }^{*} P=0.012$ ), and similar to mice receiving $C s f 1 r^{-/}$grafts (Figure 6D), M279-treated mice showed no evidence of cutaneous fibrosis 
A

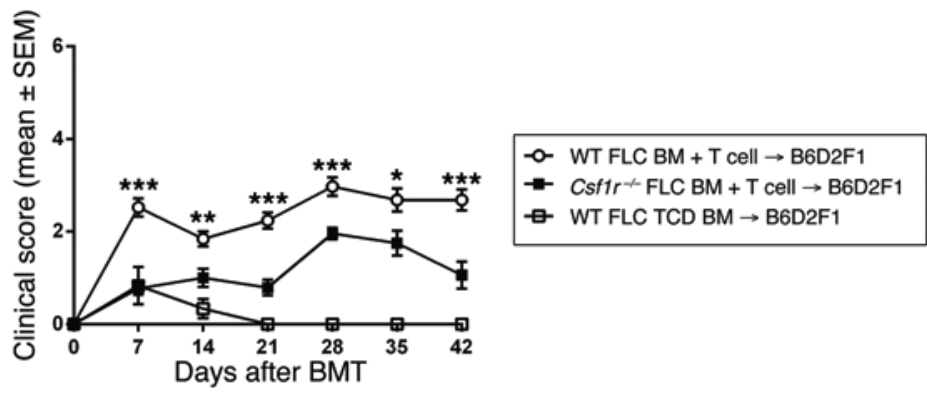

B
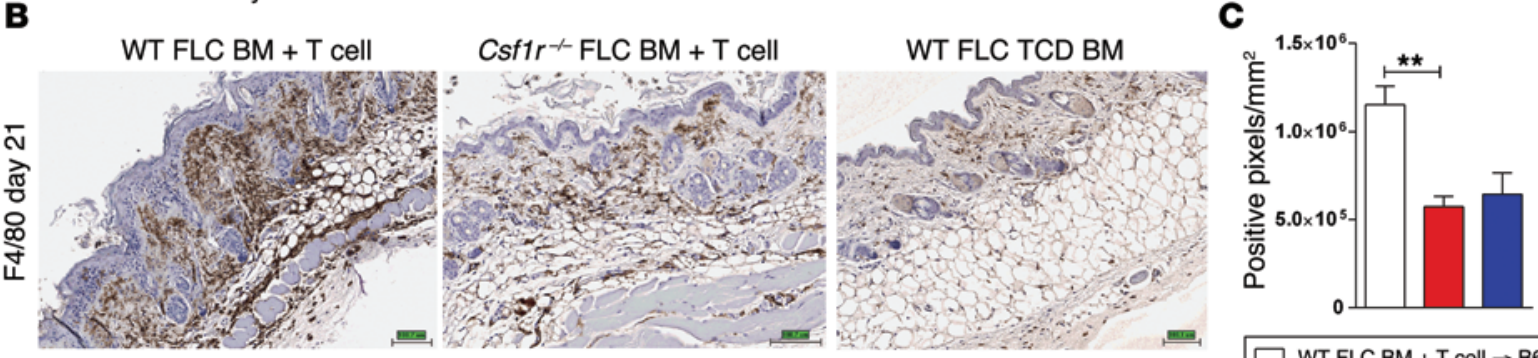

D
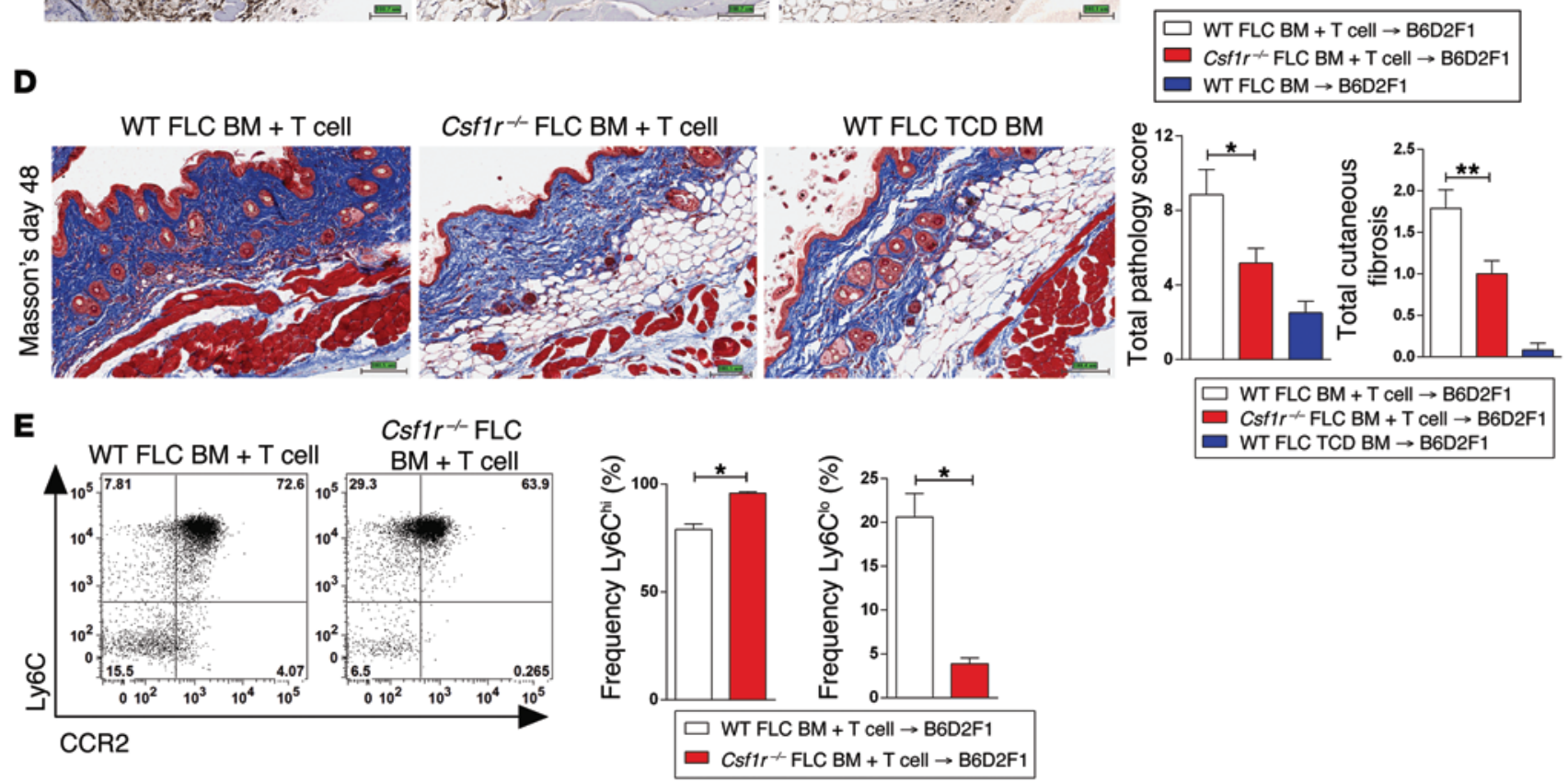

$\square$ WT FLC BM + T cell $\rightarrow$ B6D2F1 $\square$ Csf1 $r^{-1} \mathrm{FLC} \mathrm{BM}+\mathrm{T}$ cell $\rightarrow \mathrm{B} 6 \mathrm{D} 2 \mathrm{~F} 1$ WT FLC TCD BM $\rightarrow$ B6D2F1

Figure 6. F4/80+ macrophage infiltration and cutaneous fibrosis following BMT are CSF-1/CSF-1R dependent. Lethally irradiated B6D2F1 mice received BM plus T cells from WT FLCs or Csf1r ${ }^{-1-}$ FLCs (KO FLCs). (A) Mice that received KO FLC grafts had significantly lower GVHD clinical scores (day 7, ${ }^{* * *} P \leq 0.0001$; day $14,{ }^{* *} P=0.007$; day $21,{ }^{* * *} P=0.0001$; day $28,{ }^{* *} P=0.0003$; day $35,{ }^{*} P=0.043$ ). (B) IHC for $F 4 / 80$ expression from skin 21 days after transplantation

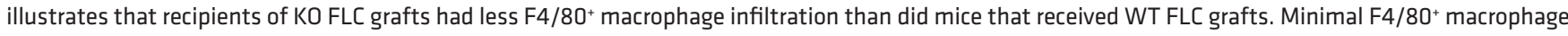
infiltrate was noted in mice that received TCD grafts. (C) Quantification of F4/80+ staining as positive/pixels $/ \mathrm{mm}^{2}\left({ }^{*} P=0.003\right)$. (D) Masson's trichrome images and semiquantitative histopathology ( $n=19 \mathrm{WT}, n=23 \mathrm{KO}, n=6 \mathrm{TCD}$ ) scores for cutaneous pathology and total cutaneous fibrosis on day 48 after transplantation confirmed that recipients of KO FLC grafts had significantly lower fibrosis compared with those that received WT FLC grafts $\left({ }^{*} P=0.045\right.$; $\left.{ }^{* *} P=0.008\right)$. (E) Representative dot plots of PB monocyte/macrophage analysis of recipients 21 days after transplantation. Numbers in each dot plot indicate the percentage of Ly6C hi cells (top 2 quadrants) and Ly6C ${ }^{10}$ cells (bottom 2 quadrants) and their expression of CCR2. Results illustrate a significant increase in the frequency of Ly6C $C^{\text {hi }}$ cells and a significant decrease in Ly6 ${ }^{10}$ cell frequency in recipients of KO FLC grafts $\left({ }^{*} P=0.0286,{ }^{*} P=0.0286\right) . n=4 /$ group. Statistically significant differences were calculated using 2-tailed Mann-Whitney $U$ tests. Data represent the mean \pm SEM. Original magnification, $\times 5$.

(Figure 9D; $\left.{ }^{* *} P=0.0006\right)$. PB analysis on day 48 after transplantation also confirmed that M279 mAb treatment resulted in a significant increase in the frequency of Ly6 $\mathrm{C}^{\text {hi }}$ monocytes and a significant decrease in Ly6C ${ }^{\text {lo }}$ monocytes (Figure 9E; ${ }^{* *} \mathrm{P}=0.0012$, $\left.{ }^{\star} P=0.0262\right)$. Thus, CSF-1R Ab-mediated depletion of donor macrophages after transplantation may be an effective therapeutic strategy to prevent and/or treat cutaneous cGVHD.
Development of cutaneous GVHD is TGF- $\beta$ dependent. We have previously reported that in the $\mathrm{B} 10 . \mathrm{BR} \rightarrow \mathrm{BALB} / \mathrm{c}$ model of scleroderma, skin-infiltrating $\mathrm{CD} 11 \mathrm{~b}^{+}$mononuclear cells produce high levels of TGF- $\beta$, and neutralization of this cytokine attenuated cGVHD (31). We therefore speculated that TGF- $\beta$ is a mediator of fibrotic skin pathology invoked by macrophages in the current study. Thus, we examined the expression of TGF- $\beta$ in the CD11b ${ }^{+} \mathrm{F} 4 / 80^{+} \mathrm{Ly} 6 \mathrm{C}^{\text {lo }}$ 


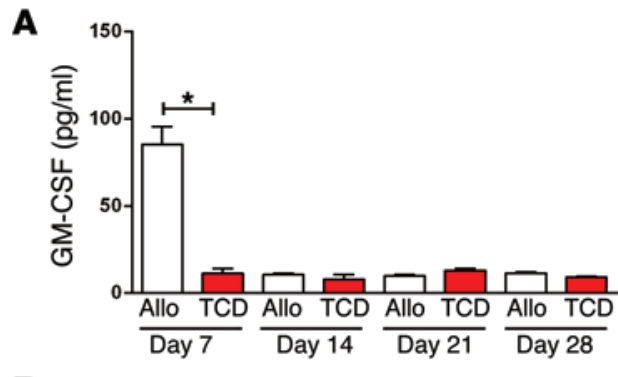

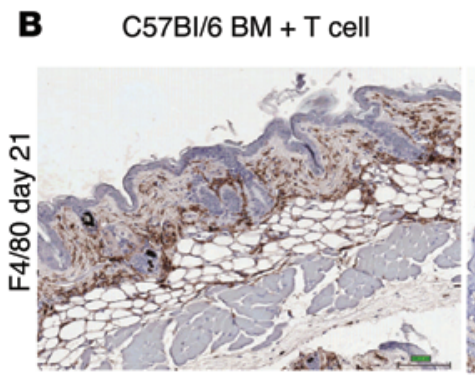
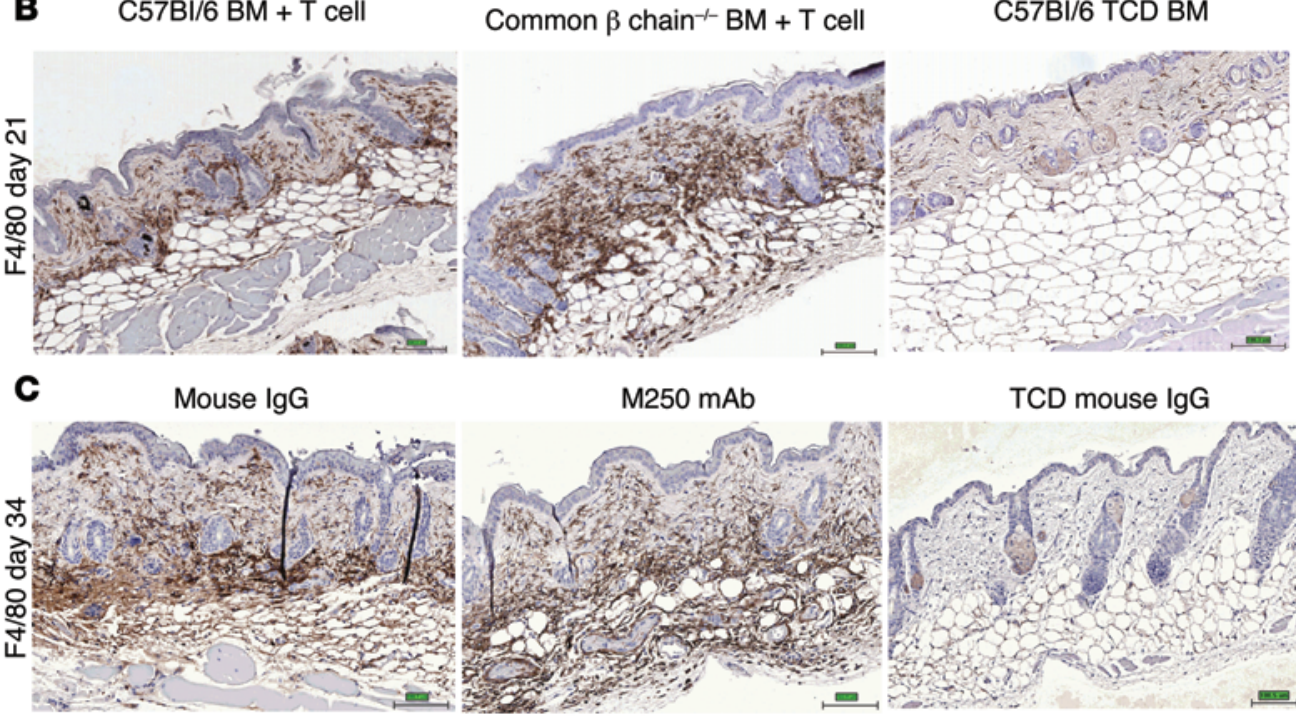

M250 mAb

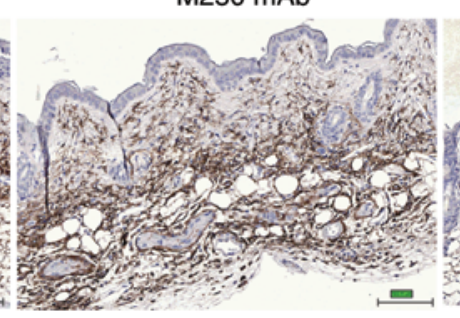

TCD mouse IgG
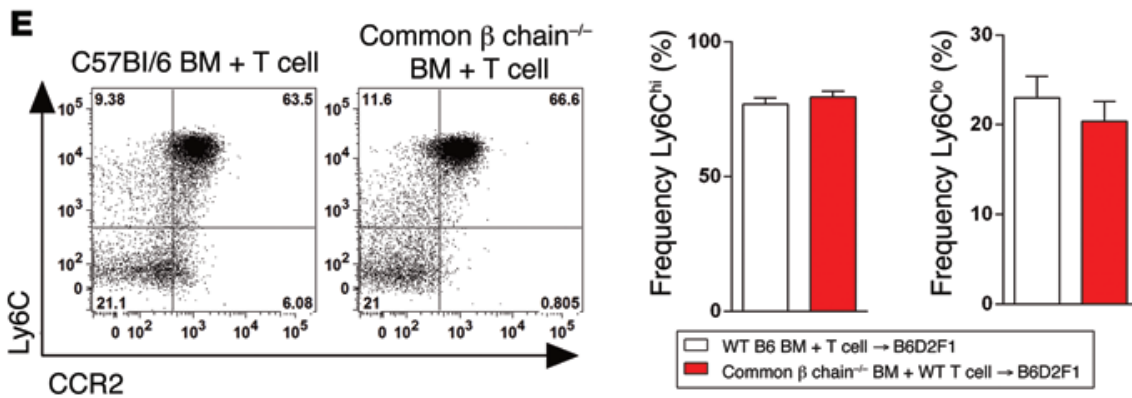

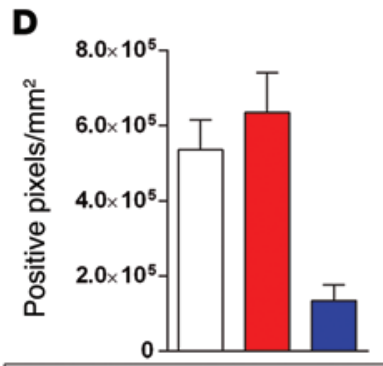

$\square$ G-CSF BALB/C $\rightarrow$ C57BI mouse IgG $\square$ G-CSF BALB/C $\rightarrow$ C57BV/6 M250 mAb - G-CSF BALB/C TCD $\rightarrow$ C57BI/6 mouse lgG

Figure 7. F4/80+ macrophage infiltration and cutaneous fibrosis after BMT is GM-CSF/GM-CSFR independent. (A) GM-CSF serum levels (pg/ml) in lethally irradiated B6D2F1 mice that received either B6 BM plus T cell (Allo) or TCD grafts 7, 14, 21, and 28 days after transplantation. (B) Representative images of IHC to detect F4/80 expression illustrate that recipients of common $\beta$ chain ${ }^{-1-}$ grafts still acquired $\mathrm{F} 4 / 80^{+}$macrophage infiltration after transplantation ( $n=6 \mathrm{WT}, n=4 \mathrm{KO}, n=3 \mathrm{TCD}$ ). (C) Lethally irradiated B6 mice received either G-CSF-mobilized BALB/c grafts or TCD grafts and were treated with anti-GM-CSF (400 $\mu$ g every 2 weeks) from days 7 to 33 after transplantation. Representative IHC images of F4/80 expression at day 34 after transplantation ( $n=4$ /group for all groups, except 3/TCD group), confirming that M250 treatment did not reduce F4/80 macrophage infiltration, quantified in $\mathbf{D}$ as positive/pixels $/ \mathrm{mm}^{2}$. (E) Representative dot plots of $\mathrm{PB}$ monocyte/macrophage analysis of recipients 21 days after transplantation. Numbers in each dot plot indicate the percentage of Ly6C ${ }^{\text {hi }}$ cells (top 2 quadrants) and Ly6C ${ }^{10}$ cells (bottom 2 quadrants) and their expression of CCR2. Results illustrate no significant changes in either Ly6C ${ }^{\text {hi }}$ or Ly $6 C^{10}$ cell frequencies between groups ( $n=4 /$ group). Statistically significant differences were calculated using 2-tailed Mann-Whitney $U$ tests. Data represent the mean \pm SEM. Original magnification, $\times 5$.

PB monocyte precursor population after transplantation. Our results confirmed that the PB monocyte precursors to the pathogenic tissue macrophages rapidly produced TGF- $\beta$ in response to TLR4 ligation with LPS (Supplemental Figure 4A). Furthermore, Ab blockade of TGF- $\beta$ from days 14 to 46 after transplantation significantly reduced GVHD clinical scores and, importantly, significantly decreased cutaneous pathology compared with the control mAb-treated group (Supplemental Figure 4, B and C). Taken together, the data suggest that donor CSF-1R-dependent macrophages contribute to CGVHD via the expression of TGF- $\beta$.
Development of lung GVHD is IL-17 and CSF-1R dependent. Both transplantation models used above (G-CSF-mobilized BALB/c $\rightarrow$ $\mathrm{B} 6$ and $\mathrm{B} 6 \mathrm{BM}$ plus $\mathrm{T}$ cell $\rightarrow \mathrm{B} 6 \mathrm{D} 2 \mathrm{~F} 1$ ) resulted in the development of scleroderma after transplantation, but neither model elicited fibrotic manifestations in other cGVHD target organs. Therefore, we next investigated the contribution of CSF-1/CSF-1R-dependent macrophages to cGVHD using the recently described multiorgan system cGVHD model (B6 $\rightarrow$ B10.BR) (32), in which lung injury is associated with obstructive lung disease and fibrosis, with increased collagen deposition surrounding bronchioles (bronchiolitis obliterans 


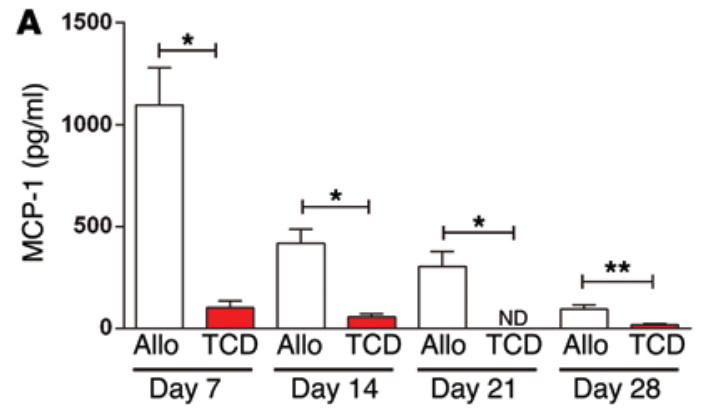

B $\quad B 6 B M+T$ cell

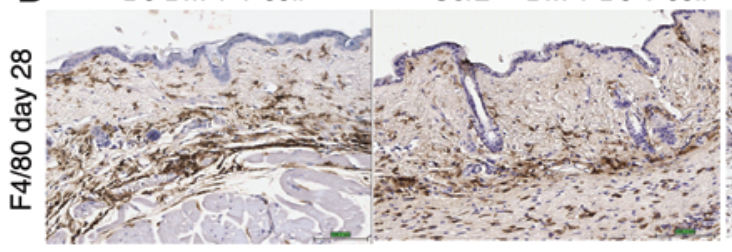

D $\quad \mathrm{B} 6 \mathrm{BM}+\mathrm{T}$ cell
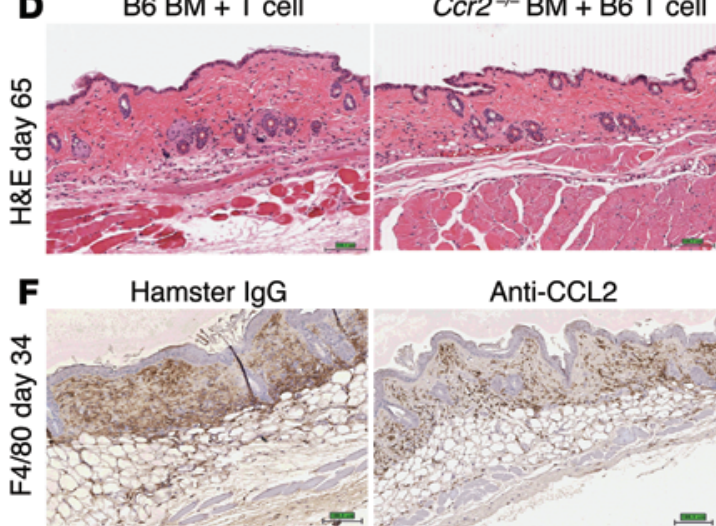

Anti-CCL2
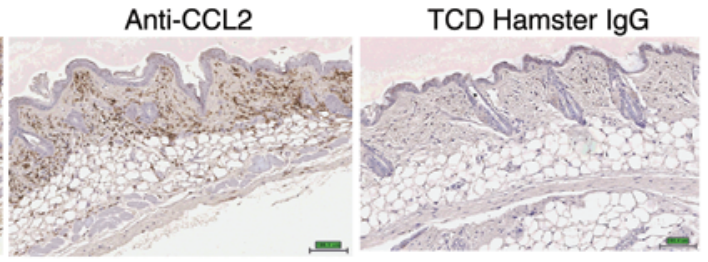

B6 BM TCD

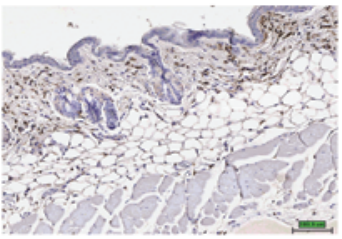

B6 BM TCD

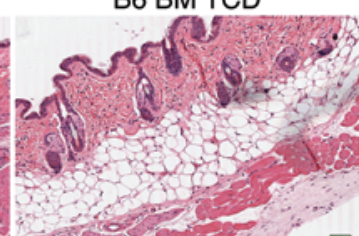

C

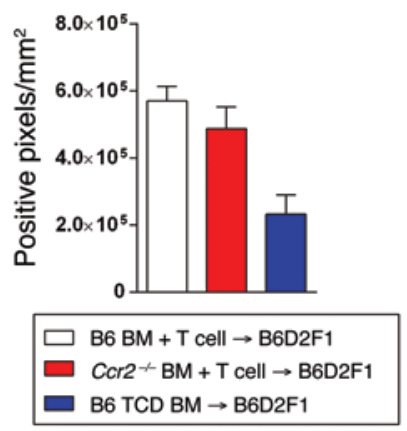

E
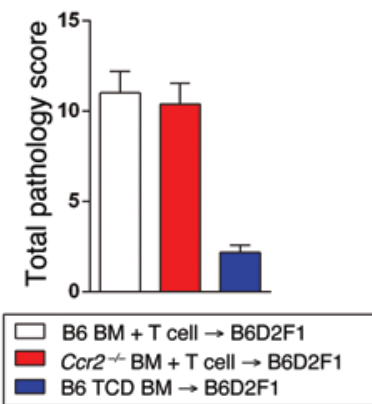

G

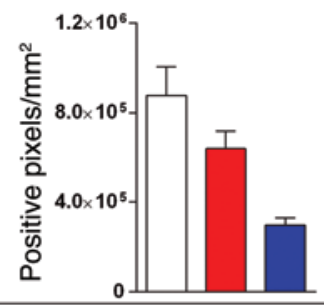

$\square \mathrm{G}-\mathrm{CSF} \mathrm{BALB} / \mathrm{C} \rightarrow \mathrm{B} 6$ hamster $\mathrm{lgC}$

G-CSF BALB/c $\rightarrow$ B6 Anti-CCL2

G-CSF BALB/c TCD $\rightarrow$ B6 hamster lgG

H

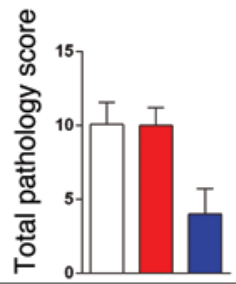

G-CSF BALB/c $\rightarrow$ C57BI/6 hamster IgG $\square \mathrm{G}-\mathrm{CSF}$ BALB/c $\rightarrow$ C57BI/6 Anti-CCL2 - G-CSF BALB/C TCD $\rightarrow$ C57BI/6 hamster IgG
I

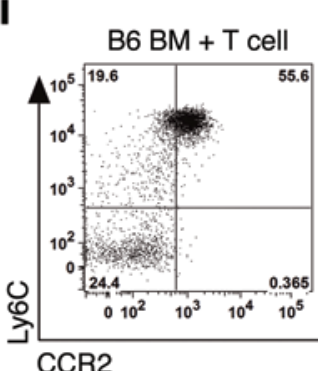

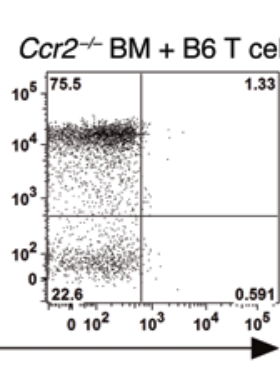

Figure 8. $\mathbf{F 4 / 8 0 ^ { + }}$ macrophage infiltration and cutaneous fibrosis post BMT is CCL2/CCR2 independent. (A) Serum CCL2 (MCP-1; pg/ml) in lethally irradiated B6D2F1 mice that received B6 BM plus T cell (Allo) or TCD grafts 7, 14, 21, and 28 days after transplantation (days 7 and $14,{ }^{*} P=0.015$; day $21,{ }^{*} P=0.035$; day $\left.28,{ }^{* *} P=0.009\right) . n=4-6 /$ group. (B) Lethally irradiated B6D2F1 mice received BM plus $T$ cell grafts from B6 or $C c r 2^{-/-}$donors. IHC to detect F4/80 expression at day 28 shows that recipients of $C \mathrm{Cr}^{-1-}$ grafts had F4/80+ macrophage infiltration levels similar to those in mice that received WT grafts, quantified in $\mathbf{C}$ as positive/pixels $/ \mathrm{mm}^{2}(P=0.207)(n=9 \mathrm{WT}, n=8 \mathrm{KO}, n=5 \mathrm{TCD})$. (D and E) H\&E-stained images and semiquantitative histopathology ( $n=15 \mathrm{WT}, n=14$ KO, $n=6$ TCD) illustrating that recipients of $C \mathrm{Cr}^{-1-}$ grafts had cutaneous pathology similar to that of recipients of WT grafts $(P=0.720)$. (F and $\left.\mathbf{G}\right)$ Lethally irradiated B6 mice received G-CSF-mobilized BALB/C or TCD grafts and were treated with hamster IgG or anti-CCL2 from days 7 to 33 ( $n=15$ hamster IgG, $n=14$ anti-CCL2, $n=3$ TCD). Similar levels of F4/80+ infiltration were noted in both groups, quantified in $\mathbf{G}$ as positive $/ \mathrm{pixels}^{2} / \mathrm{mm}^{2}(P=0.2727)$. (H) Semiquantitative histopathology score illustrating that anti-CCL2 administration had no effect on cutaneous pathology $(P=0.922)$. (I) Representative dot plots of recipient PB monocyte and macrophage analysis at day 21. Numbers in each dot plot indicate the percentage of Ly6Chi cells (top 2 quadrants) and Ly6Clo cells (bottom 2 quadrants) and their expression of CCR2. Results show no significant changes in Ly6C ${ }^{\text {hi }}$ or Ly6C ${ }^{10}$ cell frequencies ( $n=4 /$ group). Statistically significant differences were calculated using 2-tailed Mann-Whitney $U$ tests. Data represent the mean \pm SEM. Original magnification, $\times 5$.

[BO]) but without scleroderma manifestations. $\mathrm{BO}$ is characterized by airway blockage, peribronchiolar fibroproliferation, and obliteration of bronchioles and is a late-stage complication of GVHD. Pulmonary function tests (PFTs) demonstrated that recipients of BM plus splenocyte (Sp) grafts that developed cGVHD also had elevated airway resistance, which correlated with lung constriction (Resistance), elevated elastance, which signifies increased stiffness or rigidity of the lungs (Elastance), and decreased total lung capacity 
A

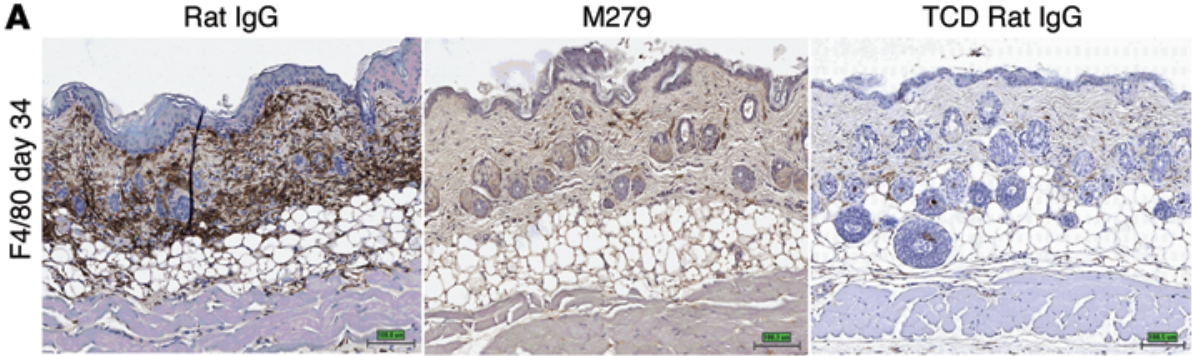

C Rat $\lg G$

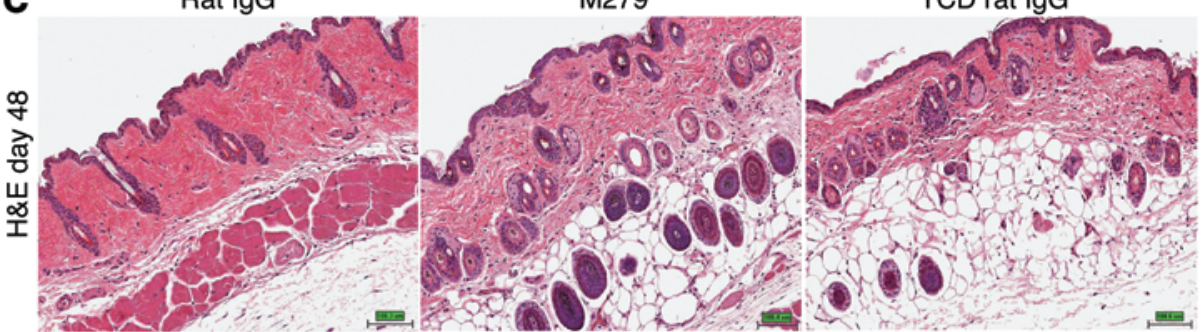

B

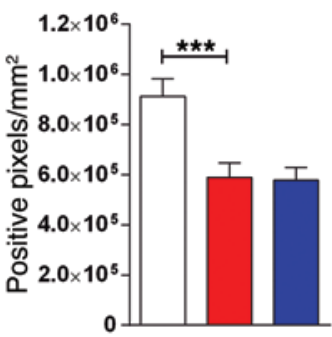

-CSF BALB/C $\rightarrow$ B6 rat lgG G-CSF BALB/C $\rightarrow$ B6 M279 G-CSF BALB/C TCD $\rightarrow$ B6 rat lgG

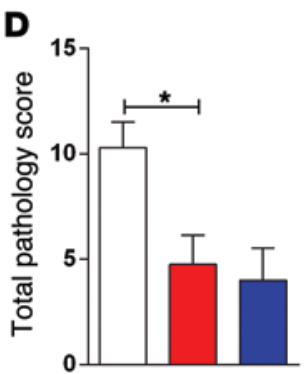

E
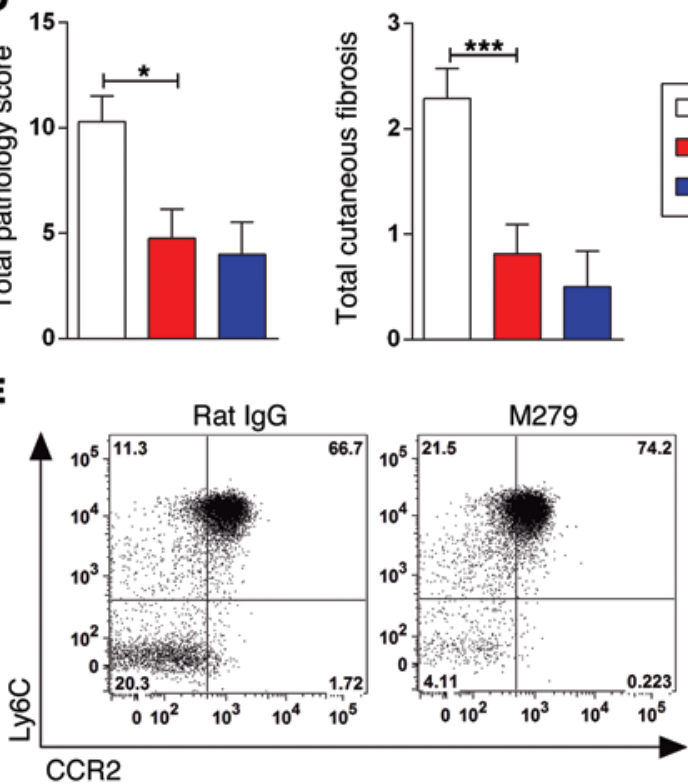

$\mathrm{B} 6 \mathrm{BM}+\mathrm{T}$ cell $\rightarrow \mathrm{B} 6 \mathrm{D} 2 \mathrm{~F} 1$ rat $\mathrm{lgG}$

$\mathrm{B} 6 \mathrm{BM}+\mathrm{T}$ cell $\rightarrow \mathrm{B} 6 \mathrm{D} 2 \mathrm{~F} 1 \mathrm{M} 279$

B6 TCD BM $\rightarrow$ B6D2F1 rat IgG
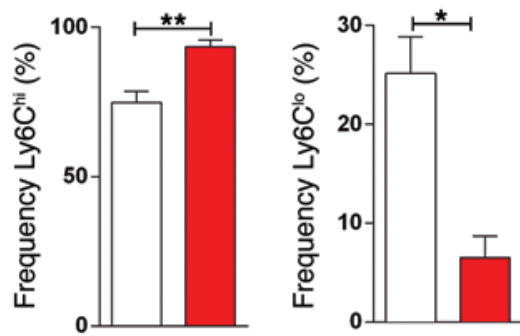

$\square \mathrm{B} 6 \mathrm{BM}+\mathrm{T}$ cell $\rightarrow \mathrm{B} 6 \mathrm{D} 2 \mathrm{~F} 1$ rat IgG $\mathrm{B} 6 \mathrm{BM}+\mathrm{T}$ cell $\rightarrow \mathrm{B} 6 \mathrm{D} 2 \mathrm{~F} 1 \mathrm{M} 279$

Figure 9. Anti-CSF-1R Ab treatment after transplantation attenuates cutaneous GVHD. Lethally irradiated B6 mice received G-CSF-mobilized WT BALB/C grafts and were treated with rat IgC control or anti-CSF-1R mAb (M279; $400 \mu \mathrm{g} / 3$ times week) from days 7 to 33 after transplantation. (A) IHC to detect F4/80 expression 34 days after transplantation ( $n=8-10$ /group for all groups; $n=3 /$ group for TCD), confirming that M279 treatment resulted in a significant depletion of $\mathrm{F} 4 / 80^{+}$cells, quantified in $\mathbf{B}$ as positive/pixels $/ \mathrm{mm}^{2}\left({ }^{* *} P=0.002\right)$. (C and $\left.\mathbf{D}\right)$ Lethally irradiated B6D2F1 mice received B6 BM and T cell grafts and were treated with either rat IgG or M279 from days 7 to 48 after transplantation ( $n=7-8 /$ group; $n=3 /$ group for TCD). (C and D) H\&E-stained images and semiquantitative histopathology confirmed that M279 treatment resulted in a significant reduction in cutaneous pathology and cutaneous fibrosis ( ${ }^{*} P=0.01 ;{ }^{* *} P=0.0006$ ). (E) Representative dot plots of PB monocyte and macrophage analysis of recipients 48 days after transplantation. Numbers in each dot plot indicate the percentage of Ly6C hi cells (top 2 quadrants) and Ly6C ${ }^{\text {lo }}$ cells (bottom 2 quadrants) and their expression of CCR2. Results show a significant increase in the frequency of Ly6C ${ }^{\text {hi }}$ cells and a significant decrease in Ly $6{ }^{10}$ cell frequencies in M279-treated recipients $\left({ }^{* *} P=0.0012 ;{ }^{*} P=0.026\right) . n=7 /$ group. Statistically significant differences were calculated using 2-tailed Mann-Whitney $U$ tests. Data represent the mean \pm SEM. Original magnification, $\times 5$.

and compliance (Compliance) (32). As with cutaneous GVHD (9), lung GVHD developed in an IL-17-dependent manner, since mice receiving grafts deficient in the retinoid-related orphan receptor $\gamma$ $\left(\mathrm{RorC}^{-/}\right)$, which is required for lineage commitment in IL-17-producing cells, exhibited significantly better pulmonary function relative to that of mice receiving WT grafts (Figure 10A). We acknowledge that we cannot discriminate the cellular sources of IL-17 by this approach and that effects may be mediated by T cells, innate lymphoid cells, or another as-yet uncharacterized cytokine-producing cell population. Development of lung pathology was also confirmed to be CSF-1R

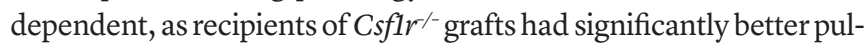
monary function compared with that of mice receiving WT grafts or those receiving WT BM with Csflr/- splenocytes (Figure 10B). Recipients of $\mathrm{Csfl}^{--} \mathrm{BM}$ plus splenocyte grafts also displayed a significant 
A Resistance

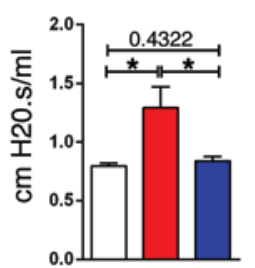

B

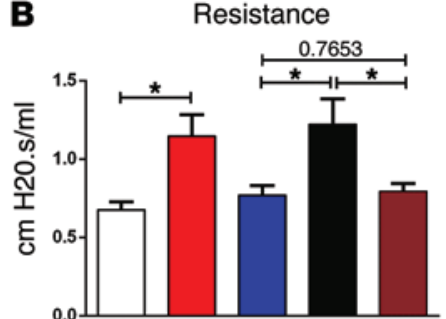

Elastance

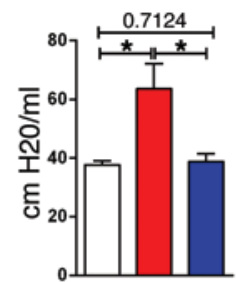

Elastance

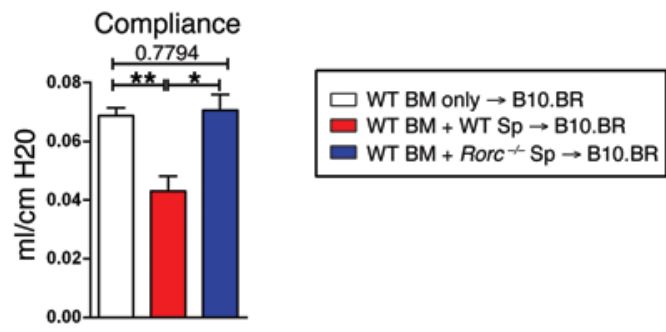

Compliance

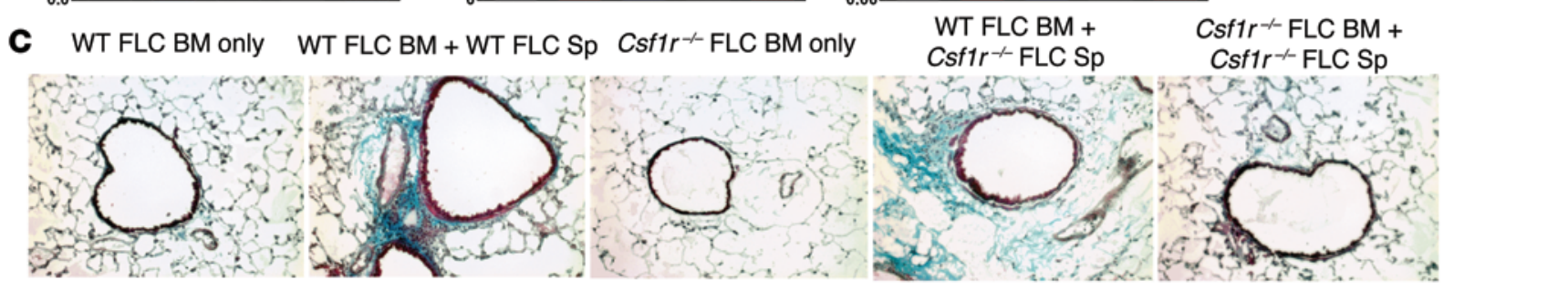

D
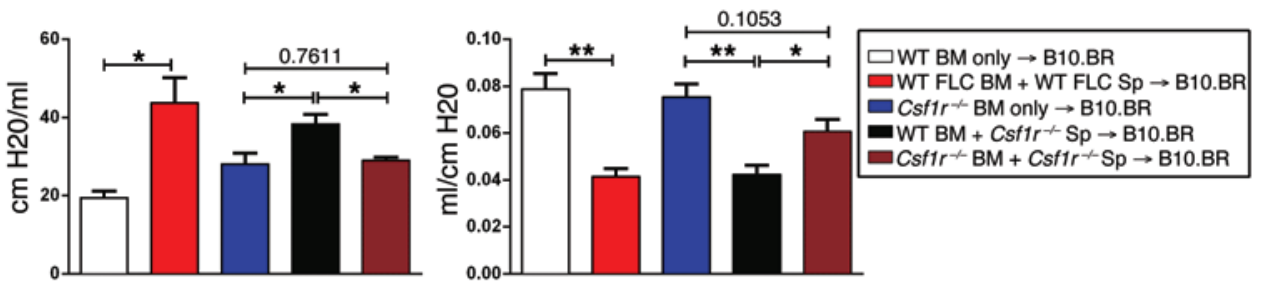

C WT FLC BM only WT FLC BM + WT FLC Sp Csf1r $r^{-1-}$ FLC BM only

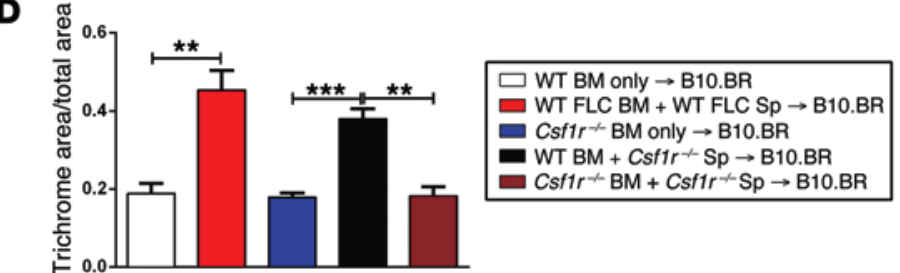

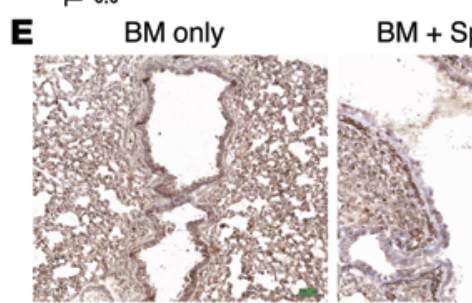

G

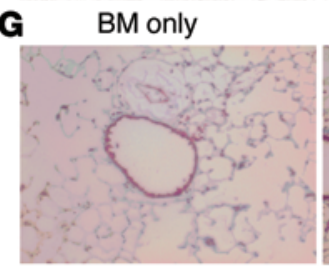

$B M+S p$ Rat $\lg G$

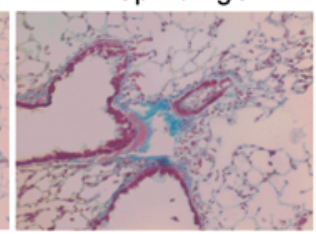

$\mathrm{BM}+\mathrm{Sp}$ M279

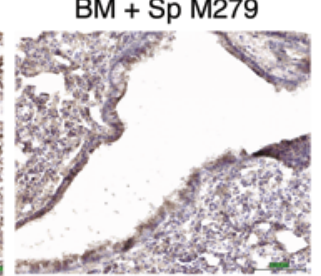

BM + Sp M279
F Resistance

Elastance
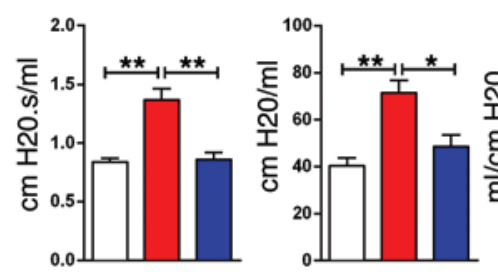

$\square$ B6 BM only $\rightarrow$ B10.BR
B6 BM + T cell $\rightarrow$ B10.BR control IgG B6 BM + T cell $\rightarrow$ B10.BR M279

H

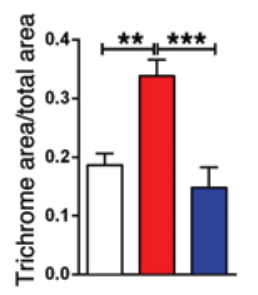

Compliance

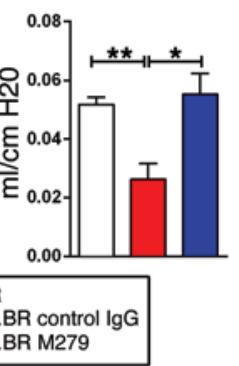

$\square$ B6 BM + T cell $\rightarrow$ B10.BR control IgG B6 BM + T cell $\rightarrow$ B10.BR M279

Figure 10. Lung GVHD develops in an IL-17- and CSF-1R-dependent manner. (A) B10.BR recipients treated with $120 \mathrm{mg} / \mathrm{kg} / \mathrm{day}$ cyclophosphamide (days -3 and -2 ) and lethally irradiated (day -1; 850 cGy) were transplanted with B6 BM with either B6 or Rorc ${ }^{-/-} \mathrm{Sp}(n=4 /$ all groups). On day 60 after transplantation, pulmonary function measures were performed. R, resistance; E, elastance; C, compliance. Recipients of Rorc ${ }^{-/-}$grafts exhibited significantly improved pulmonary function compared with that of WT graft recipients ( $\mathrm{R}:{ }^{*} P=0.02,{ }^{*} P=0.03 ; \mathrm{E}:{ }^{*} P=0.01,{ }^{*} P=0.03 ; \mathrm{C}:{ }^{* *} P=0.005$, ${ }^{*} P=0.01$ ). (B) Similarly, recipients of $C s f 1 r^{-1-}$ BM plus $C s f 1 r^{-1-}$ Sp grafts had significantly improved pulmonary function compared with that of mice receiving WT BM plus Csf1r ${ }^{-1}$ Sp. (R: $\left.{ }^{*} P=0.024,{ }^{*} P=0.042,{ }^{*} P=0.0431 ; \mathrm{E}:{ }^{*} P=0.013,{ }^{*} P=0.031,{ }^{*} P=0.01 ; \mathrm{C}:{ }^{*} P=0.001,{ }^{* *} P=0.0031,{ }^{*} P=0.033\right) . n=4$. (C) Trichrome staining illustrates reduced collagen deposition in mice that received $C s \mathrm{f}^{-/-}$grafts compared with those that received WT grafts, quantified in $\mathbf{D}$ as trichrome area/total area $\left.{ }^{* *} P=0.0037 ;{ }^{* *} P=0.0005 ;{ }^{* *} P=0.0023\right)$. (E) IHC for $\mathrm{F} 4 / 80$ expression in recipients of WT B6 plus Sp or BM-only grafts that were given control IgC or M279 mAb after transplantation from days 0 to 28 after transplantation. Minimal F4/80+ cells were noted in recipients treated with M279 $\mathrm{mAb}$ compared with those detected in control IgG-treated recipients. (F) Lung function parameters confirmed an improvement in lung function after M279 mAb treatment $\left(\mathrm{R}:{ }^{* *} P=0.001,{ }^{* *} P=0.008 ; \mathrm{E}:{ }^{* *} P=0.003,{ }^{*} P=0.034 ; \mathrm{C}:{ }^{* *} P=0.004,{ }^{*} P=0.02\right) . n=4$. (C) Trichrome staining confirmed that M279 mAb treatment significantly reduced collagen deposition, quantified in $\mathbf{H}\left({ }^{* *} P=0.017 ;{ }^{* *} P=0.0003\right)$. Statistically significant differences were calculated using unpaired $t$ tests. Data represent the mean \pm SEM. Original magnification, $\times 20$. 
reduction in collagen deposition around the bronchioles compared with recipients of WT grafts (Figure 10C), quantified as trichrome area/total area (Figure 10D). These data suggest that donor BM is the source of CSF-1 $\mathrm{R}^{+}$tissue-resident macrophages that are essential for cGVHD-induced BO.

Last, we investigated whether M279 mAb treatment resulted in a reduction in lung cGVHD. Recipients were administered control IgG or M279 mAb after transplantation from days 0 to 28 . IHC to detect $\mathrm{F} 4 / 80$ expression confirmed that macrophages infiltrated the lungs of mice that received BM plus Sp grafts and rat IgG (Figure $10 \mathrm{E}$ ), with reduced $\mathrm{F} 4 / 80^{+}$cell infiltration in recipients treated with M279 mAb. We noted minimal macrophage infiltration in mice that received BM-only grafts (Figure 10E). Furthermore, we found that M279 mAb treatment significantly improved all lung PFT parameters (Figure 10F) and resulted in a significant reduction in collagen deposition (Figure 10G), quantified as trichrome area/ total area (Figure 10H). Overall, these data confirm that cutaneous and pulmonary GVHD develop in a CSF-1/CSF-1R-dependent manner, and depletion of this population after transplantation represents a novel therapeutic strategy to prevent and treat cGVHD.

\section{Discussion}

Macrophages have been shown to play crucial roles in tissue repair as well in the resolution and/or progression of tissue fibrosis (10-13). This study provides new insight into the pathogenic role of donor macrophages in the progression of fibrosis, the cardinal feature of cGVHD. We specifically demonstrate that CSF-1/CSF-1R-dependent donor BM-derived macrophages infiltrate GVHD target organs as early as 7 days after transplantation, which precedes the development of cutaneous and pulmonary GVHD. The infiltration of these macrophages was demonstrated to be both CCR2 and GM-CSF independent. Most importantly, blocking the action of CSF-1/CSF-1R using either $\mathrm{Cs} \mathrm{flr}^{-1-}$ mice or an anti-CSF-1R Ab depleted these donor macrophages from the skin and lung, resulting in a dramatic reduction in cGVHD.

The origin of tissue-resident macrophages remains controversial. During development, yolk sac and liver embryonic macrophage precursors seed the resident macrophage populations of most tissues including the skin, liver, and lung. During the neonatal period, these tissue-resident macrophage populations are expanded by local proliferation and maintained during adulthood by self-renewal (33). In the gut, however, the resident macrophage population is derived from $\mathrm{BM}(34,35)$. During injury or infection, PB monocytes can be recruited to tissues and sites of inflammation and give rise to a variety of tissue-resident macrophages. In mice, circulating monocyte populations can be divided into 2 distinct groups (22). The classical/inflammatory monocytes, which are classified as $\mathrm{Ly}_{6 \mathrm{C}}{ }^{\mathrm{hi}}, \mathrm{CCR} 2^{+}$, and $\mathrm{Cx} 3 \mathrm{CR} 1^{\mathrm{lo}}$, constitute the monocyte subset most likely to be recruited to sites of inflammation. The nonclassical/patrolling monocytes are Ly6 $\mathrm{C}^{\mathrm{lo} /}, \mathrm{CCR}^{\mathrm{lo} /}{ }^{\mathrm{l}}$, and $\mathrm{Cx} 3 \mathrm{CR} 1^{+}$and are thought to contribute to tissue-resident macrophage populations $(22,36)$. Whether the patrolling monocytes give rise to tissue macrophages under steady state has recently come into question $(37,38)$. Importantly, during fibrosis, at least in the liver, macrophages are suggested to be derived from either tissue-resident cell populations, such as Kupffer cells, or from BM immigrants $(39,40)$. Although SCT induces a highly inflammatory environment including an early systemic release of MCP-1 and GM-CSF, our data surprisingly illustrate that neither CCR2 nor GM-CSF signaling is required for the development of cutaneous GVHD and that, instead, fibrosis in both skin and lung models of GVHD is CSF-1 dependent. Blocking the action of CSF-1/CSF-1R using either $\mathrm{Csflr}^{--}$mice or an anti-CSF-1R Ab resulted in a significant decrease in $\mathrm{Ly} 6 \mathrm{C}^{\text {lo }}$ cell populations in both the blood and skin. As previously observed (30), the reduction in the Ly6 $\mathrm{C}^{\text {lo }}$ cell population was balanced by an increase in the $\mathrm{Ly} 6 \mathrm{C}^{\mathrm{hi}}$ cell population, supporting the proposed model that suggests a precursor relationship between the immature inflammatory monocytes and mature resident monocytes in vivo $(36,41)$. Therefore, we propose that the precursor to the pathogenic tissue macrophages that sequester in the GVHD target organs is a nonclassical/patrolling F4/80 ${ }^{+} \mathrm{CD}_{11 \mathrm{~b}^{+} \text {Ly6C }} \mathrm{C}^{\mathrm{lo}}$ donor BM-derived monocyte subset.

Macrophages and CSF-1/CSF-1R signaling have already been shown to contribute to engraftment and the development of aGVHD following transplantation (42). CSF-1 levels are substantially elevated in the circulation in mice with aGVHD, which could function to extend the survival of host macrophages after transplantation (43). We previously demonstrated that pretreatment of recipients with the anti-CSF-1R mAb M279 prior to allogeneic BMT significantly ablated macrophages in GVHD target organs, resulting in exaggerated donor $\mathrm{T}$ cell activation and accelerated GVHD pathology after transplantation (30). Furthermore, host intestinal macrophages were also shown to engulf and clear donor $\mathrm{T}$ cells, thus contributing to the attenuation of alloreactive $\mathrm{T}$ cell responses (44). Therefore, donor and host macrophages appear to exert opposing effects on GVHD outcomes, with host macrophages being protective for aGVHD, while donor macrophages are pathogenic for cGVHD. Thus, in contrast to the attenuation of cGVHD that CSF-1 blockade provided, interrupting this pathway in the early peritransplantation period could exacerbate aGVHD. These observations are reminiscent of our earlier findings, in which TGF- $\beta$ neutralization early after transplantation resulted in a significant exacerbation of aGVHD, whereas delayed neutralization attenuated sclerodermatous cGVHD (31). In that study, a skin-infiltrating donor $\mathrm{CD}_{11} \mathrm{~b}^{+}$mononuclear cell population expressed the highest levels of TGF- $\beta$. Here, we confirm and extend these findings, demonstrating that blockade of TGF- $\beta$ after transplantation in the $\mathrm{B} 6 \rightarrow \mathrm{B} 6 \mathrm{D} 2 \mathrm{~F} 1$ model significantly reduced cutaneous pathology and further demonstrating TGF- $\beta$ expression by the $\mathrm{F} 4 / 8 \mathrm{O}^{+} \mathrm{CD} 11 \mathrm{~b}^{+} \mathrm{Ly} 6 \mathrm{C}^{\text {lo }}$ blood monocyte precursor to the pathogenic skin macrophages. Taken together, the data support the notion that donor CSF-1-dependent skin-infiltrating macrophages contribute to cutaneous GVHD in a TGF- $\beta$-dependent manner.

These studies highlight Ly $6 \mathrm{C}^{\text {lo }}$ monocyte precursors, macrophages, and their effector molecules (e.g., TGF- $\beta$ ) as potential targets for the prevention and treatment of cGVHD. In the current study, we have used a mAb against mouse CSF-1R that blocks the actions of CSF-1 without inducing apoptosis or overt inflammation (30). While it is clear that blockage of CSF-1R signaling can have functional consequences on tissue-resident macrophages (45), treatment with the anti-CSF-1R mAb M279 following transplantation phenocopied the effects produced using a $\mathrm{Cs} \mathrm{flr}^{-/-}$allograft (i.e., a dramatic reduction in skin-infiltrating macrophages and attendant pathology). While we can- 
not completely exclude additional effects on function, the M279 $\mathrm{mAb}$ appears to be acting on monocytes and macrophages in more of a quantitative rather than a qualitative fashion when used after BMT. There are multiple agents that target the CSF$1 \mathrm{R} / \mathrm{CSF}-1$ pathway in clinical trials, predominantly to ablate tumor-associated macrophages as a means to limit tumor progression. These include a humanized version of the rodent CSF$1 \mathrm{R}$ mAb used here. In addition, many small-molecule tyrosine kinase inhibitors such as imatinib, sunitinib, and sorafenib are used in clinical practice to treat various malignancies. Interestingly, imatinib, at least, has significant off-target effects that interrupt the CSF-1R pathway (46), and this agent has demonstrated efficacy, albeit limited, in the treatment of cGVHD (4749). Together, it appears that targeting the CSF-1 pathway may provide an effective means for treating chronic GVHD, and clinical trials to test this hypothesis could now be rapidly initiated.

\section{Methods}

Mice. Female C57Bl/6 (B6) (H-2b, CD45.2), B6.Ptprc ${ }^{\mathrm{a}}\left(\mathrm{H}-2^{\mathrm{b}}, \mathrm{CD} 45.1^{+}\right)$, $\mathrm{BALB} / \mathrm{c}(\mathrm{H}-2 \mathrm{~d}, \mathrm{CD} 45.2)$, and B6D2F1 (H-2b/d, CD45.2) mice were purchased from the Animal Resources Center. Mice were housed in sterilized microisolator cages and received acidified autoclaved water ( $\mathrm{pH}$ 2.5) after transplantation. The mice ranged in age from 8 to 14 weeks. BALB/c CD45.1 (H-2 $^{\mathrm{d}}$ ) mice were supplied by Mark Smyth (QIMR Berghofer Medical Research Institute, Queensland, Australia). $\mathrm{Csfl}^{-/+}$mice were provided by Richard E. Stanley (Albert Einstein College of Medicine, New York, New York, USA). C-C chemokine receptor $2\left(\mathrm{Cr}^{-/}\right)$(50) mice were provided by Mark Smyth (QIMR Berghofer Medical Research Institute). Common $\beta$ chain $^{-/-}\left(C s f 2 r b^{\text {tmlcgh }}\right)(24)$ mice were provided by Angel Lopez (Center for Cancer Biology, Ade-

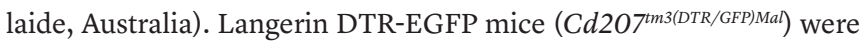
provided by Bernard Malissen (Centre d'Immunologie de Marseille, Luminy, France) (51). B10.BR (H-2k) mice were purchased from the Jackson Laboratory, and B6 $\left(\mathrm{H} 2^{\mathrm{b}}\right)$ mice were purchased from the National Cancer Institute.

Cytokine mobilization. Recombinant human G-CSF was administered s.c. at $10 \mu \mathrm{g} / \mathrm{animal}$ daily for 6 days.

Generation of Csf1r/- FLCs. To generate FLCs, mice heterozygous for Csfir deficiency were time-mated, and at E18/19, pregnant mice were culled, pups removed, and the fetal liver harvested into a single-cell suspension. A portion of each pup's tail was harvested into QuickExtract DNA extraction solution (Epicentre Biotechnologies) for genomic DNA extraction, and PCR was performed on each to determine both the Csfir genotype (WT, heterozygote, or homozygote) and the sex of each pup. Primer sequences for Csfir genotyping were as follows: CSF-1R in exon 2, forward: 5'-AGACTCATTCCAGAACCAGAGC-3'; CSF-1R in exon 3, reverse: 5'-GAATTTGGAGTCCTCACCTTTG-3'; and CSF-1R neo, forward: 5'-CCGGTAGAATTCCTCGAGTCTA-3'. Primer sequences for sex differentiation genotyping were as follows: $\mathrm{X}$ chromosome, forward: 5'-GTTCTTTCGTTTTCCCCTCTC-3'; X chromosome, reverse: 5'-GGCATTATCTAAGGAGAAGCATC-3'; Y chromosome (a): 5'-GACTAGACATGTCTTAACATCTGTCC-3'; and Y chromosome (b): 5'-CCTATTGCATGGACAGCAGCTTATG-3'. Female CSF-1R-KO and WT pups were identified, and $2 \times 10^{6}$ FLCs from each were injected separately into lethally irradiated (1,000 cGy) PTPRCA mice. These mice were left for 3 months to allow recon- stitution of the immune system, and mice were confirmed to have donor engraftment greater than $98 \%$ before being used as transplant donors or recipients.

SCT and BMT. On day -1 , mice received $1,000 \mathrm{cGy}$ (B6) or 1,100 cGy (B6D2F1) total-body irradiation (137 Cs source at approximately $85 \mathrm{cGy} /$ minute), split into 2 doses separated by 3 hours to minimize gastrointestinal toxicity. On day 0 , B6 mice each received $25 \times 10^{6}$ donor Sp from G-GSF-mobilized BALB/c donors. Sp were depleted of T cells as previously described (52) for non-GVHD control groups. In BMT experiments, B6D2F1 recipients underwent transplantation on day 0 with $5 \times 10^{6} \mathrm{BM}$ with or without $1 \times 10^{6}$ purified T cells. Mice received $\mathrm{BM}$ from either B6, common $\beta$ chain $^{-/}$, Ccr2 ${ }^{-/}$, WT FLC, Csfr1 $1^{-1-}$ FLC, or langerin DTR-EGFP mice. Non-GVHD control groups were injected with TCD BM grafts. Transplanted mice were monitored daily, and those with GVHD clinical scores greater than or equal to $6(53,54)$ were killed and the date of death registered as the next day in accordance with institutional animal ethics committee guidelines. Recipients of langerin DTR-EGFP grafts were treated with saline or DT $(0.1 \mu \mathrm{g} / \mathrm{mouse}$; Sigma-Aldrich) twice weekly from days 7 to 40 . For Ab-mediated depletion, from 7 days after SCT, mice received either $500 \mu$ g every 2 weeks of mouse IgG or anti-GM-CSFR (M250; Amgen) or $400 \mu \mathrm{g} 3$ times per week of anti-CSF-1R Ab (M279; Amgen) or rat IgG control $\mathrm{Ab}$ - all via i.p. injection. For TGF- $\beta$ blockade, from 7 days after transplantation, mice were treated with either control or antiTGF- $\beta$ Ab (in-house, ATCC) $(20 \mu \mathrm{g} / 3$ times per week) via i.p. injection. B10.BR recipients were conditioned with cyclophosphamide on days -3 and $-2(120 \mathrm{mg} / \mathrm{kg} /$ day i.p.). On day -1 , mice received lethal irradiation (850 cGy). On day 0 , mice received $1 \times 10^{7} \mathrm{BM}$ cells from either WT B6, WT FLC, or Csf1 $1 r^{-/}$FLC mice with $1 \times 10^{6}$ Sp from either WT B6 or Csflr ${ }^{-1}$ B6 mice or from Rorc $^{-/}$B6 mice.

Assessment of GVHD. The degree of systemic GVHD was assessed using a cumulative scoring system that measures changes based on 5 clinical parameters: weight loss, posture (hunching), activity, fur texture, and skin integrity (maximum index 10). Mice were monitored daily, and those with GVHD clinical scores (53) greater than or equal to 6 were culled and the date of death recorded as the next day in accordance with institutional animal ethics guidelines.

Fluorescence-activated cell-sorting analysis. The following antibodies were purchased from BioLegend: phycoerythrin-conjugated (PE-conjugated) anti-F4/80, allophycocyanin-conjugated anti-CCR2, PE-Cy7-conjugated anti-IA/IE, APC-Cy7-conjugated anti-Ly6G, Pacific Blue-conjugated anti-Ly6, and PerCP-Cy5.5-conjugated CD11b. PE.CF594-conjugated CD3 was purchased from BD Biosciences. Biotinylated anti-TGF- $\beta$ was purchased from R\&D Systems.

Tissue digests. For ear skin digests, the ears were removed and each ear split into dorsal and ventral surfaces with forceps. Skin was floated on $2 \mathrm{ml}$ of dispase solution (Gibco) and incubated for 2 hours at $37^{\circ} \mathrm{C}$. Dermal and epidermal sheets were detached with forceps and transferred to $2 \mathrm{ml}$ of a 0.1- $\mathrm{mg} / \mathrm{ml}$ collagenase solution (Sigma-Aldrich) in HBSS (Mediatech) with $\mathrm{Ca}^{++}$supplemented with $10 \% \mathrm{FBS}$, and $250 \mu \mathrm{l}$ of 20,000 U/ml DNAse I (Roche) per 50-ml working solution. Skin was cut into small pieces and incubated for 1.5 hours at $37^{\circ} \mathrm{C}$. Epidermal and dermal cells were disaggregated by pipetting 10 times with a syringe and needle (19-gauge), filtered through nylon mesh to remove clumps, and the mesh was washed with flow buffer to reduce cell loss $(1 \times$ PBS/ethylenediaminetetraacetic acid, $2 \mathrm{mM}$ BSA $0.5 \%)$, then centrifuged for 5 minutes at $400 \mathrm{~g}$. 
$P B$ in vitro stimulation. Unfractionated red cell-lysed PB was cultured with 100 ng LPS for 2 hours in Iscove's modified Dulbecco's medium (IMDM) supplemented with 10\% FBS (Gibco), $1 \mathrm{mM}$ sodium pyruvate (Thermo Scientific), $1 \mathrm{mM}$ Pen Strep (Gibco), $1 \mathrm{mM}$ glutamine (HyClone), $1 \mathrm{mM}$ nonessential amino acids (Gibco), and $23 \mathrm{mM}$ $\beta$-mercaptoethanol (Sigma-Aldrich).

Histopathology and immunostaining of GVHD target organs. At various times after transplantation, GVHD target tissues were harvested, fixed in $10 \%$ formalin for 24 hours, embedded in paraffin, and processed to generate 5 - $\mu \mathrm{m}$-thick sections. All lung tissue harvested was embedded in optimal cutting-temperature compound, snap-frozen in liquid nitrogen, and stored at $-80^{\circ} \mathrm{C}$. Lungs were inflated by infusion of $1 \mathrm{ml}$ of optimal cutting-temperature compound/PBS (3:1) intratracheally before harvesting. H\&E sections of skin were examined in a blinded fashion (by A.D. Clouston) using a semiquantitative scoring system for GVHD as previously published (54). Samples were scored from 0 to 4 for epidermal and dermal inflammation, dermal fibrosis and subcutaneous fibrosis (summed to give a total cutaneous fibrosis score of 8), epidermal apoptosis (total score of 4), and loss of s.c. fat (total score of 4). IHC was performed on deparaffinized and rehydrated sections. Briefly, slides were incubated with antigen retrieval solution (0.37\% Carezyme Trypsin; Biocare Medical) for 10 minutes. Slides were then incubated in serum block for 60 minutes (10\% FBS, 10\% normal goat serum), followed by rat anti-mouse $\mathrm{F} 4 / 80 \mathrm{Ab}$ (BioLegend) or rat IgG2b isotype-matched control for 60 minutes. Endogenous peroxidase activity was blocked using $3 \% \mathrm{H}_{2} \mathrm{O}_{2}$. Cells were subsequently incubated with a goat anti-rat biotinylated $\mathrm{F}\left(\mathrm{ab}^{\prime}\right)_{2}$ fragment (Santa Cruz Biotechnology Inc.), followed by HRP-conjugated streptavidin (Jackson ImmunoResearch). DAB was developed according to the manufacturer's instructions (Dako), and all slides were counterstained with Mayer's hematoxylin and mounted using permanent mounting media.

For trichrome staining, 6- $\mu \mathrm{m}$ sections were cut and incubated in Bouin's solution for 15 minutes at $55^{\circ} \mathrm{C}$, washed, and stained for 5 minutes in Weigert's iron hematoxylin solution. Slides were washed in tap water, then distilled water for 5 minutes and stained for 5 minutes in Biebrich Scarlet-Acid Fuchsin solution (Thermo Scientific). Slides were subsequently washed in distilled water, stained in phosphotungstic/ phosphomolybdic acid solution for 5 minutes, aniline blue solution for 5 minutes, and $1 \%$ acetic acid solution for 2 minutes, and washed in distilled water to remove all residue. Slides were then dehydrated through alcohol baths and xylene washes and coverslipped. Images were collected using a BX51 light microscope (Olympus).

Frozen tissues issues were embedded in Tissue-Tek OCT compound (Sakura Finetek) without prior fixation, and IHC was carried out on $7-\mu \mathrm{m}$ sections fixed with $75 \%$ acetone $/ 35 \%$ ethanol. IHC was performed as described above but with the addition of an endogenous avidin/biotin blocking step (10 minutes with avidin, 10 minutes with biotin; Vector Labs). Slides were then blocked in serum as described above [with an additional $\mathrm{F}\left(\mathrm{ab}^{\prime}\right.$ ) fragment-blocking step for CD45.1 Ab IHC](Jackson ImmunoResearch) and incubated in one of the following primary antibodies: rat anti-mouse F4/80 (BioLegend), rat anti-mouse CD206 (AbD Serotec); rabbit anti-mouse iNOS (Abcam), or mouse anti-mouse CD45.1 (BioLegend) or matching isotype controls, and normal Rabbit IgG (Santa Cruz Biotechnology Inc.) or mouse IgG (BioLegend). Endogenous peroxidase activity was blocked using $3 \% \mathrm{H}_{2} \mathrm{O}_{2}$. Cells were subsequently incubated with either goat anti-rat or goat anti-rabbit biotinylated $\mathrm{F}\left(\mathrm{ab}^{\prime}\right)_{2}$ fragment followed by HRP-conjugated streptavidin. Slides were developed as described above. Immunofluorescence (IF) of frozen tissues was performed as described above, but using streptavidin-conjugated Alexa Fluor 555 tertiary Ab. Slides were then stained with DAPI. All IHC slides were viewed using an Aperio Scanscope XT microscope with Scanscope software (version 10.2.2.2352). IF slides were viewed on an LSM 710 confocal microscope (Zeiss) with Image $1.44 \mathrm{p}$ software (NIH).

PFTs. PFTs were performed as described previously (55). Anesthetized mice were weighed, and lung function was assessed by whole-body plethysmography using the Flexivent system (SCIREQ) and analyzed with Flexivent version 5.1 software.

Cytokine analysis. Serum cytokine concentrations were determined using the BD Cytometric Bead Array system (BD Biosciences - Pharmingen) according to the manufacturer's protocol.

Statistics. Survival curves were plotted using Kaplan-Meier estimates and compared by log-rank analysis using PRISM 5 (GraphPad Software). A $P$ value of less than 0.05 was considered statistically significant. A 2-tailed Mann-Whitney $U$ test or an unpaired $t$ test was used to evaluate significant differences between groups, and all data represent the mean \pm SEM.

Study approval. All mouse experiments were performed in accordance with and under the approval of the QIMR Berghofer Medical Research Institute's Animal Ethics Committee and the IACUC of the University of Minnesota.

\section{Acknowledgments}

K.A. Markey is a National Health and Medical Research Council Clinical Training Fellow. M. Koyama is a Leukemia Foundation of Australia Postdoctoral Fellow. G.R. Hill is a National Health and Medical Research Council Australia Fellow and Queensland Health Senior Clinical Research Fellow. K.P.A. MacDonald is a Cancer Council Queensland Senior Research Fellow. This work was supported by the National Health and Medical Research Council of Australia (ID496689) and by NIH grants P01 CA142106 and P01 AI 056299 (to B.R. Blazer) and T32 AI007313 (to R. Flynn).

Address correspondence to: Kelli MacDonald, Antigen Presentation and Immunoregulation Laboratory, QIMR Berghofer Medical Research Institute, 300 Herston Road, Brisbane, QLD 4006, Australia.Phone: 61.7.3362.0404; E-mail: Kelli.MacDonald@qimr.edu.au.
1. Bensinger WI, et al. Transplantation of bone marrow as compared with peripheral-blood cells from HLA-identical relatives in patients with hematologic cancers. $N$ Engl J Med. 2001;344(3):175-181.

2. Gordon S, Taylor PR. Monocyte and macrophage heterogeneity. Nat Rev Immunol. 2005;5(12):953-964.
3. Bonifer C, Hume DA. The transcriptional regulation of the Colony-Stimulating Factor 1 Receptor (csflr) gene during hematopoiesis. Front Biosci. 2008;13:549-560.

4. Stanley ER, et al. Biology and action of colony - stimulating factor-1. Mol Reprod Dev. 1997;46(1):4-10.

5. Gordon S. Alternative activation of macrophages.
Nat Rev Immunol. 2003;3(1):23-35.

6. Mantovani A, Sica A, Sozzani S, Allavena P, Vecchi A, Locati M. The chemokine system in diverse forms of macrophage activation and polarization. Trends Immunol. 2004;25(12):677-686

7. Hamilton JA. Colony-stimulating factors in inflammation and autoimmunity. Nat Rev Immunol. 
2008;8(7):533-544.

8. Stein M, Keshav S, Harris N, Gordon S. Interleukin 4 potently enhances murine macrophage mannose receptor activity: a marker of alternative immunologic macrophage activation. JExp Med.1992;176(1):287-292.

9. Hill GR, et al. Stem cell mobilization with G-CSF induces type 17 differentiation and promotes scleroderma. Blood. 2010;116(5):819-828.

10. Duffield JS, et al. Selective depletion of macrophages reveals distinct, opposing roles during liver injury and repair. J Clin Invest. 2005;115(1):56-65.

11. Pesce JT, et al. Arginase-1-expressing macrophages suppress Th2 cytokine-driven inflammation and fibrosis. PLoS Pathog. 2009;5(4):e1000371.

12. Murray LA, et al. TGF-beta driven lung fibrosis is macrophage dependent and blocked by Serum amyloid P. Int J Biochem Cell Biol. 2011;43(1):154-162.

13. Ko GJ, Boo CS, Jo SK, Cho WY, Kim HK. Macrophages contribute to the development of renal fibrosis following ischaemia/reperfusion-induced acute kidney injury. Nephrol Dial Transplant. 2008;23(3):842-852.

14. Wang $\mathrm{H}$, et al. Activated macrophages are essential in a murine model for $\mathrm{T}$ cell-mediated chronic psoriasiform skin inflammation. JClin Invest. 2006;116(8):2105-2114.

15. Stratis A, et al. Pathogenic role for skin macrophages in a mouse model of keratinocyteinduced psoriasis-like skin inflammation. JClin Invest. 2006;116(8):2094-2104.

16. Nishiwaki S, et al. Impact of macrophage infiltration of skin lesions on survival after allogeneic stem cell transplantation: a clue to refractory graft-versus-host disease. Blood. 2009;114(14):3113-3116.

17. Shimizu K, et al. Increased serum levels of soluble CD163 in patients with scleroderma. Clin Rheumatol. 2012;31(7):1059-1064.

18. Juniantito $\mathrm{V}$, et al. Immunophenotypical characterization of macrophages in rat bleomycin-induced scleroderma. Vet Pathol. 2013;50(1):76-85.

19. Higashi-Kuwata N, et al. Characterization of monocyte/macrophage subsets in the skin and peripheral blood derived from patients with systemic sclerosis. Arthritis Res Ther. 2010;12(4):R128.

20. Sun L, et al. New concepts of IL-10-induced lung fibrosis: fibrocyte recruitment and M2 activation in a CCL2/CCR2 axis. Am J Physiol Lung Cell Mol Physiol. 2011;300(3):L341-L353.

21. Sasmono RT, et al. A macrophage colony-stimulating factor receptor-green fluorescent protein transgene is expressed throughout the mononuclear phagocyte system of the mouse. Blood. 2003;101(3):1155-1163.

22. Geissmann F, Jung S, Littman DR. Blood monocytes consist of two principal subsets with distinct migratory properties. Immunity. 2003;19(1):71-82.

23. Greter M, et al. Stroma-derived interleukin-34 controls the development and maintenance of langerhans cells and the maintenance of microglia. Immunity. 2012;37(6):1050-1060.
24. Robb L, et al. Hematopoietic and lung abnormalities in mice with a null mutation of the common $\beta$ subunit of the receptors for granulocyte-macrophage colony-stimulating factor and interleukins 3 and 5. Proc Natl Acad Sci U S A. 1995;92(21):9565-9569.

25. Lloyd CM, Dorf ME, Proudfoot A, Salant DJ, Gutierrez-Ramos JC. Role of MCP-1 and RANTES in inflammation and progression to fibrosis during murine crescentic nephritis. J Leukoc Biol. 1997;62(5):676-680.

26. Hasegawa M, Sato S, Takehara K. Augmented production of chemokines (monocyte chemotactic protein-1 (MCP-1), macrophage inflammatory protein-1alpha (MIP-1 $\alpha$ ) and MIP-1 $\beta$ ) in patients with systemic sclerosis: MCP- 1 and MIP- $1 \alpha$ may be involved in the development of pulmonary fibrosis. Clin Exp Immunol. 1999;117(1):159-165.

27. Distler JH, et al. Monocyte chemoattractant protein 1 released from glycosaminoglycans mediates its profibrotic effects in systemic sclerosis via the release of interleukin- 4 from $\mathrm{T}$ cells. Arthritis Rheum. 2006;54(1):214-225.

28. Yamamoto T, Eckes B, Hartmann K, Krieg T. Expression of monocyte chemoattractant protein-1 in the lesional skin of systemic sclerosis. J Dermatol Sci. 2001;26(2):133-139.

29. Scala E, et al. Cytokine and chemokine levels in systemic sclerosis: relationship with cutaneous and internal organ involvement. Clin Exp Immunol. 2004;138(3):540-546.

30. MacDonald KP, et al. An antibody against the colony-stimulating factor 1 receptor depletes the resident subset of monocytes and tissue- and tumor-associated macrophages but does not inhibit inflammation. Blood. 2010;116(19):3955-3963.

31. Banovic T, et al. TGF- $\beta$ in allogeneic stem cell transplantation: friend or foe? Blood. 2005;106(6):2206-2214.

32. Srinivasan M, et al. Donor B-cell alloantibody deposition and germinal center formation are required for the development of murine chronic GVHD and bronchiolitis obliterans. Blood. 2012;119(6):1570-1580.

33. Ginhoux F, et al. Fate mapping analysis reveals that adult microglia derive from primitive macrophages. Science. 2010;330(6005):841-845.

34. Bogunovic M, et al. Origin of the lamina propria dendritic cell network. Immunity. 2009;31(3):513-525.

35. Varol C, et al. Intestinal lamina propria dendritic cell subsets have different origin and functions. Immunity. 2009;31(3):502-512.

36. Sunderkotter C, et al. Subpopulations of mouse blood monocytes differ in maturation stage and inflammatory response. J Immunol. 2004;172(7):4410-4417.

37. Hashimoto D, et al. Tissue-resident macrophages self-maintain locally throughout adult life with minimal contribution from circulating monocytes. Immunity. 2013;38(4):792-804.

38. Yona $\mathrm{S}$, et al. Fate mapping reveals origins and dynamics of monocytes and tissue macrophages under homeostasis. Immunity. 2013;38(1):79-91.

39. Ramadori G, Armbrust T. Cytokines in the liver. Eur J Gastroenterol Hepatol. 2001;13(7):777-784.
40. Luckey SW, Petersen DR. Activation of Kupffer cells during the course of carbon tetrachloride-induced liver injury and fibrosis in rats. Exp Mol Pathol. 2001;71(3):226-240.

41. Tacke F, Ginhoux F, Jakubzick C, van Rooijen $\mathrm{N}$, Merad M, Randolph GJ. Immature monocytes acquire antigens from other cells in the bone marrow and present them to $T$ cells after maturing in the periphery. J Exp Med. 2006;203(3):583-597.

42. Blazar BR, Aukerman SL, Vallera DA. Effect of recombinant human macrophage colony-stimulating factor in irradiated murine recipients of T-cell-depleted allogeneic or non-depleted syngeneic bone marrow transplants. Blood. 1992;79(6):1636-1642.

43. Praloran V, et al. Alterations in the expression of colony-stimulating factor- 1 and its receptor during an acute graft-vs-host reaction in mice. J Immunol.1990;145(10):3256-3261.

44. Hashimoto D, et al. Pretransplant CSF-1 therapy expands recipient macrophages and ameliorates GVHD after allogeneic hematopoietic cell transplantation. J Exp Med. 2011;208(5):1069-1082.

45. Pyonteck SM, et al. CSF-1R inhibition alters macrophage polarization and blocks glioma progression. Nat Med. 2013;19(10):1264-1272.

46. Dewar AL, et al. Macrophage colony-stimulating factor receptor c-fms is a novel target of imatinib. Blood. 2005;105(8):3127-3132.

47. Magro L, et al. Imatinib mesylate as salvage therapy for refractory sclerotic chronic graft-versushost disease. Blood. 2009;114(3):719-722.

48. Olivieri A, et al. Imatinib for refractory chronic graft-versus-host disease with fibrotic features. Blood. 2009;114(3):709-718.

49. de Masson A, et al. Limited efficacy and tolerance of imatinib mesylate in steroid-refractory sclerodermatous chronic GVHD. Blood. 2012;120(25):5089-5090.

50. Boring L, et al. Impaired monocyte migration and reduced type 1 (Th1) cytokine responses in C-C chemokine receptor 2 knockout mice. J Clin Invest. 1997;100(10):2552-2561.

51. Kissenpfennig A, et al. Dynamics and function of Langerhans cells in vivo: dermal dendritic cells colonize lymph node areas distinct from slower migrating Langerhans cells. Immunity. 2005;22(5):643-654.

52. Burman AC, et al. IFN $\gamma$ differentially controls the development of idiopathic pneumonia syndrome and GVHD of the gastrointestinal tract. Blood. 2007;110(3):1064-1072.

53. Cooke KR, et al. An experimental model of idiopathic pneumonia syndrome after bone marrow transplantation: $\mathrm{I}$. The roles of minor $\mathrm{H}$ antigens and endotoxin. Blood. 1996;88(8):3230-3239.

54. Hill GR, Crawford JM, Cooke KR, Brinson YS, Pan L, Ferrara JL. Total body irradiation and acute graft-versus-host disease: the role of gastrointestinal damage and inflammatory cytokines. Blood.1997;90(8):3204-3213.

55. Panoskaltsis-Mortari A, Tram KV, Price AP, Wendt $\mathrm{CH}$, Blazar BR. A new murine model for bronchiolitis obliterans post-bone marrow transplant. Am J Respir Crit Care Med. 2007;176(7):713-723. 